\title{
Does a central clearing counterparty reduce liquidity needs?
}

\author{
Hitoshi Hayakawa $^{1}$
}

Received: 31 March 2016 / Accepted: 8 November 2017 / Published online: 16 November 2017

(C) The Author(s) 2017. This article is an open access publication

\begin{abstract}
This study investigates whether and how central clearing influences the overall liquidity needs in a network of financial obligations. Utilizing the approach of flow network theory, we show that the effect of adding a central clearing counterparty (CCP) is decomposed into two effects: central routing, and central netting effects. Each effect can produce different liquidity needs according to different liquidity scenarios. The analysis indicates that adding a CCP in times of financial distress successfully reduces the overall liquidity needs if and only if the netting efficiency of the CCP is sufficiently high. Furthermore, once the economy is no longer in financial distress, higher netting efficiency of the CCP could conversely increase the overall liquidity needs. The results have implications for the effectiveness of CCPs in mitigating systemic risk in times of financial distress, and their operating costs once the distress has passed.
\end{abstract}

\section{Introduction}

In the recent financial crisis, the collapse of financial institutions, such as Bear Stearns and Lehman Brothers, demonstrated that the interconnected feature of bilateral exposure among financial institutions could lead to market disruption. In order to cope with this apparent vulnerability, the G20 leaders agreed at the 2009 Pittsburgh Summit that standardized over-the-counter (OTC) derivatives should be cleared through central clearing counterparties (CCPs). Central clearing is expected to help mitigate counterparty credit risk by removing the direct risk exposure between counterpar-

\footnotetext{
$凶$ Hitoshi Hayakawa

hit.hayakawa@econ.hokudai.ac.jp

1 Creative Research Institution/Graduate School of Economics and Business Administration, Hokkaido University, Kita9 Nishi7, Kita-ku, Sapporo, Japan
} 
ties, thereby reducing the systemic risk of the "domino" of defaults and relevant firesales. ${ }^{1}$

However, the success and relevant cost of central clearing is never evident. For example, CCPs typically require margin themselves in order to bear the counterparty risk arising from cleared derivative transactions. In times of financial distress, margin requirements by CCPs could trigger firesales. ${ }^{2}$ Here, we should note that multilateral netting by CCPs could have already reduced relevant exposure, and have contributed to reduce the required margin compared to settlements without CCPs. The effect of CCPs on overall liquidity needs is not apparent until the two aspects are investigated in a consolidated manner.

We further argue about the operating cost of CCPs once the economy is no longer in financial distress. CCPs could affect overall liquidity needs in times of non-distress, and larger liquidity needs tend to imply larger costs, since liquidity is essentially scarce resource. In times of non-distress, when financial institutions are not in a rush to obtain liquidity, contracted trades would need less liquidity, or similarly, liquidity would circulate more efficiently among relevant financial institutions even without CCPs. Consequently, CCPs and their multilateral netting could have different effects on overall liquidity needs compared with those in times of financial distress.

In order to assess the effects of CCPs and discuss further improvements of the clearing mechanism, it is important to understand the essential nature of CCPs regarding how they affect overall liquidity needs in times of both financial distress and nondistress. This study develops a stylized model to probe this issue. Our focus is on how the introduction of a CCP could alter the interconnected feature of the relevant network of financial obligations, and how the change of network topology could affect overall liquidity needs. From the perspective of network topology, the introduction of a CCP serves as an additional entity itself in the relevant network, while its multilateral offsetting serves to eliminate relevant obligations. We explicitly show that the effect of a CCP is decomposed into two effects: the central routing effect, and the central netting effect, such that the total effect is essentially the addition of the two effects.

The effect of a CCP is examined on the basis of two polar liquidity scenarios. One is assumed as a situation in times of financial distress, by which liquidity circulates least efficiently. The other is assumed as a benchmark situation in times of nondistress, by which liquidity circulates most efficiently. We refer to the former situation as the bad environment and the latter as the good environment. We show that in the bad environment, the central routing effect is always negative, but the central netting effect is always positive. A negative central routing effect means that adding a CCP certainly increases the overall liquidity needs if there is no financial obligation to be offset by the CCP. A positive central netting effect means that larger offset amounts lead to smaller liquidity needs. This implies that the total effect of a CCP is positive

\footnotetext{
1 In this respect, Brunnermeier and Pedersen (2009) argue there is a spiral nature between funding liquidity and market liquidity, whereby the initial loss could lead to firesales, which could further exacerbate the loss.

2 The possible procyclical feature associated with margin requirements of CCPs is pointed out in Domanski et al. (2015). Rennison et al. (2016) report a real world case that suggests the procylicality; on the day after Britain's vote for Brexit, the five of the largest clearing houses demanded \$27bn in additional collateral across derivatives products.
} 
in the bad environment when the offset amount is sufficiently large. By contrast, we show that it is possible for the central netting effect in the good environment to be negative, whereby, although counterintuitive, a larger offset amount could lead to larger liquidity needs. This is because eliminating financial obligations could effectively separate a connected network into multiple disconnected networks, thereby inhibiting the same liquidity from circulating through the whole network. We observe a tradeoff of multilateral offsetting regarding the overall liquidity needs. It is possible for a $\mathrm{CCP}$ to reduce liquidity needs during times of financial distress, thereby reducing the risk of firesales and relevant systemic risk of the "domino" of defaults. However, it could conversely increase overall liquidity needs during times of non-distress. For our benchmark situations, in order for the total effect of a CCP to be always positive in the bad environment, more than two-thirds of the relevant trading needs to be offset. Since the overall liquidity needs in the good environment could become larger in proportion to the offset amount, the trade-off could become serious.

There are two policy implications of this research. First, when central clearing is used, the expected offset efficiency for the relevant security should be examined with sufficient care, since insufficient netting efficiency could have an adverse effect. Second, possible severe trade-off associated with multilateral netting suggests conditional utilization of a CCP. Since our analysis shows that multilateral netting could be costly at the time of non-distress but helps mitigate liquidity needs at the time of financial distress, a CCP could be used as an emergency scheme. Although it is out of the scope of this study to argue about the whole cost of such an emergency scheme, our analysis indicates there is possible merit in resolving the underlying trade-off regarding overall liquidity needs.

\subsection{Relevant literature and contributions}

The role of CCPs has been examined largely focusing on how much relevant exposure is reduced through CCPs' multilateral nettings, supposing that smaller amount of exposure to each counterparty implies smaller counterparty risk. This study departs from the literature by examining the roles of CCPs in overall liquidity needs.

Focusing on the effect on exposure, Duffie and Zhu (2011) examine central clearing in derivative markets and point out the possible disadvantage of central clearing arrangements compared with bilateral clearing arrangements when central clearing is provided only within each class of derivatives. The roles of CCPs in derivative markets are debated in Bliss and Kaufman (2006), Bliss and Steigerwald (2006), and Pirrong (2009). Jackson and Manning (2007) argue about the effects of CCPs in relation to "tiering," which refers to the ratio between the number of indirect and direct members of CCPs. The authors argue there is incentive for a "tiered" structure. Galbiati and Soramäki (2012) analyze the implications of "tiering" in terms of network topology. In view of relevant network topologies, they examine the tree structure, while more general structures matter in our liquidity context.

Several recent studies have examined the role of CCPs in affecting overall liquidity needs, in the context of how CCPs set their margins. Murphy et al. (2014) investigate 
the procyclical nature of various margin models, proposing quantitative measures of procyclicality. Abruzzo and Park (2016) empirically analyze the margin-setting behavior of CCPs, and find that margin-induced procyclicality is a concern during recessions, but not during times of expansion. Miglietta et al. (2015) quantify the impact on the cost of funding in repo markets of the initial margins applied by CCPs. These studies have helped clarify the possible negative effects of CCPs on overall liquidity needs, but they have not explicitly shown how the existence of CCPs could have negative effects compared with cases without CCPs.

This study provides a stylized model that enables us to compare a situation with a $\mathrm{CCP}$ with that without a CCP. Our focus is on the effects of CCPs on overall liquidity needs by affecting the relevant network topology. For this purpose, we utilize liquidity problems defined in the network, which are formally represented as problems of flow network. The model and liquidity problems used in this study are based on Hayakawa (2016), who investigates settlement efficiency of gross settlements in view of network topology. ${ }^{3}$ The present study serves as an application of Hayakawa (2016) to examine the role of CCPs, utilizing several basic results of the study.

Section 2 presents our model. Section 3 illustrates our analysis in a less formal manner. Section 4 provides an overview of the results. Section 5 shows our formal analysis. Section 6 concludes. The appendix includes proofs of the relevant results.

\section{Model}

There are a finite number of financial institutions that have obligations among themselves. Each obligation is formed by the trade of either of two types of securities. One type is called the target security, and the other the non-target security. The target security is traded either with a CCP or bilaterally, while the non-target security is traded bilaterally. We incorporate obligations for the non-target security in order to analyze externality of the CCP in the relevant settlements. As becomes clearer soon in this section, both types of security are assumed to be of a bond or equity type in the sense that the amount of obligation formed by each trade is fixed. The simplification serves to clarify the nature of a CCP, and has implications for the debate about CCPs in derivative contracts and relevant margin issues, which are discussed in Sect. 2.4, and further in the analysis section.

We divide the process of settlements into four Stages as below.

- Stage 0: Trade formation

- Stage 1: CCP scheme/bilateral scheme

- Stage 2: Offsetting under the CCP scheme ${ }^{4}$

- Stage 3: Settlements of obligations

\footnotetext{
3 The original version of the models and liquidity problems are shown in Hayakawa (2014) with additional results, which constitutes a chapter of his doctoral thesis accepted in 2011.

4 We interchangeably use the word "offsetting" and "netting."
} 


\subsection{Stage 0: Trade formation}

At Stage 0, trades among the financial institutions are exogenously given. The contracts of the trades specify that all relevant obligations are finally settled at Stage 3. We express the obligations formed at Stage 0 utilizing a flow network representation. ${ }^{5}$

The obligations are expressed with a network $\mathcal{N}=\langle V, A, f\rangle . V$ specifies a set of vertices, which corresponds to $|V|-1$ number of the financial institutions added with one hypothetical entity. The role of the hypothetical entity is stated shortly in this section. $A=\left\{\left(v, v^{\prime}, n\right) \mid v, v^{\prime} \in V, n=1,2, \ldots\right\}$ specifies a set of arcs, where each $\operatorname{arc}\left(v, v^{\prime}, k\right)$ shows that $v$ has some amount of obligation to $v^{\prime}$, and $k$ is used as an index that distinguishes among multiple arcs for $\left(v, v^{\prime}\right)$. The indices are usually not mentioned in order to avoid being notationally cumbersome. Then, $f: A \rightarrow R_{+}$ expresses the amount of the relevant obligations. We assume that all the obligations for the trades of the target security are formed against the hypothetical entity. Using the introduced notations, $\left(\mathcal{N}=\langle V, A, f\rangle, v^{*} \in V\right)$ is exogenously given at Stage 0 , which we call a financial system with hypothetical entity $v^{*}$.

We confine ourselves to a balanced network throughout this study. We say a network $\langle V, A, f\rangle$ is balanced when for each vertex $v \in V$, the total amount of obligations owed by $v$ and those owed to $v$ are equal, that is, $\sum_{v^{\prime} \in V} f\left(\left(v^{\prime}, v\right)\right)=\sum_{v^{\prime} \in V} f\left(\left(v, v^{\prime}\right)\right)$ for every $v \in V$. This is explicitly stated as Assumption 1. The assumption of balanced networks has dual roles. On the one hand, it implies market clearing regarding the trades of the target security, since the total amount of obligations owed to and by the hypothetical entity are supposed to be equal. On the other hand, a balanced network at Stage 0 derives a balanced network at later stages. There, we examine relevant liquidity needs based on each network, and the assumption of the balanced network serves to simplify our analysis. ${ }^{6}$

Assumption 1 Given a financial system $\left(\mathcal{N}=\langle V, A, f\rangle, v^{*} \in V\right)$, network $\mathcal{N}$ is balanced.

We further assume that each of the obligations owed to and by the hypothetical entity is the same amount, that is, the price of the target security is supposed to be fixed, and each trade is made in the same unit. The assumption is formally stated as follows.

Assumption 2 Given a financial system $\left(\mathcal{N}=\langle V, A, f\rangle, v^{*} \in V\right)$, network $\mathcal{N}$ has the common obligation value $m>0$ regarding the hypothetical entity $v^{*}$ such that,

i) (obligations owed by $\left.v^{*}\right) f\left(\left(v^{*}, v\right)\right)=m$, for every $v \in V \operatorname{such}$ that $\left(v^{*}, v\right) \in A$, and

\footnotetext{
5 For basic terminologies of flow networks, we obey the textbook usages. See, for example, Ahuja et al. (1993).

6 Our analysis on balanced networks could be extended to networks that are not balanced. For a nonbalanced network, we could derive a balanced network by "local changes," such as adding arcs and/or adjusting relevant weights. Conversely, we derive the original non-balanced network through the relevant "reverse" local changes on the balanced network. The amount of liquidity needs for the original non-balanced network could be derived by examining the effects of those "reverse" local changes on the balanced network.
} 


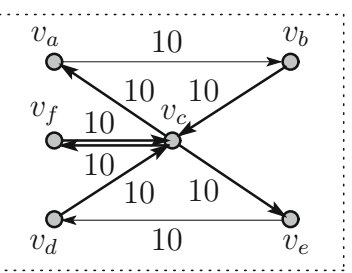

Fig. 1 Example financial system consistent with all the assumptions. Financial system $\left(\langle V, A, f\rangle, v_{c}\right)$ : $V=\left\{v_{a}, v_{b}, v_{c}, v_{d}, v_{e}, v_{f}\right\}, A=\left\{\left(v_{a}, v_{b}\right),\left(v_{b}, v_{c}\right),\left(v_{c}, v_{a}\right),\left(v_{c}, v_{e}\right),\left(v_{e}, v_{d}\right),\left(v_{d}, v_{c}\right)\right.$, $\left.\left(v_{c}, v_{f}\right),\left(v_{f}, v_{c}\right)\right\}, f(a)=10$ for every $a \in A$
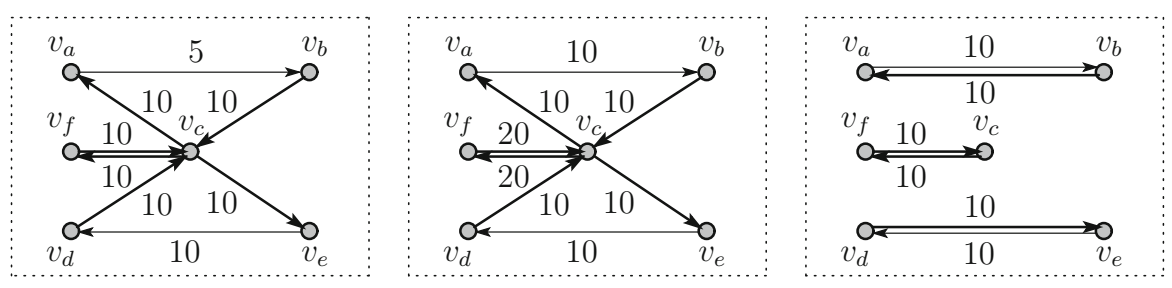

Fig. 2 Example financial systems that do not satisfy one of the assumptions. In all three financial systems, $v_{c}$ is assumed as the hypothetical entity. Each financial system violates one of the assumptions. The left financial system violates Assumption 1, since $v_{a}$ receives 10 in total, which is larger than the total amount of its payment 5. The middle financial system violates Assumption 2, since $f\left(\left(v_{f}, v_{c}\right)\right) \neq f\left(\left(v_{b}, v_{c}\right)\right)$. The right financial system violates Assumption 3, since it indicates no trade of the target security other than those by $v_{f}$

ii) (obligations owed to $\left.v^{*}\right) f\left(\left(v^{\prime}, v^{*}\right)\right)=m$, for every $v^{\prime} \in V$ such that $\left(v^{\prime}, v^{*}\right) \in A$.

Combined with Assumptions 1 and 2, the next assumption ensures there are some feasible bilateral trades for the target security, that is, if one financial institution is to buy (sell) a certain amount of the target security, at least the same amount of the target security are sold (bought) by the other financial institutions.

Assumption 3 Given a financial system $\left(\mathcal{N}=\langle V, A, f\rangle, v^{*} \in V\right)$,

i) for every $v \in V \backslash v^{*}$, the number of "buys" of the target security by $v$ is equal to or less than the number of "sells" of the target security by the other financial institutions. Formally, $\left|\left\{\left(v, v^{*}, k\right) \in A \mid k=1,2, . .,\right\}\right| \leq \mid\left\{\left(v^{*}, v^{\prime}, k\right) \in A \mid k=\right.$ $\left.1,2, . ., v^{\prime} \in V, v^{\prime} \neq v\right\} \mid$.

ii) for every $v \in V \backslash v^{*}$, the number of "sells" of the target security by $v$ is equal to or less than the number of "buys" of the target security by the other financial institutions. Formally, $\left|\left\{\left(v^{*}, v, k\right) \in A \mid k=1,2, . .,\right\}\right| \leq \mid\left\{\left(v^{\prime}, v^{*}, k\right) \in A \mid k=\right.$ $\left.1,2, \ldots, v^{\prime} \in V, v^{\prime} \neq v\right\} \mid$.

An example financial system that satisfies all Assumptions 1, 2, and 3 is shown in Fig. 1, where $v_{c}$ corresponds to the hypothetical entity, and there are six obligations for the target security. For presentational purposes, obligations for the target security are shown with thicker lines, while those for the non-target security are shown with thinner lines, and this applies throughout this article. For clarification of the assumptions, Fig. 2 shows three financial systems, in which each financial system is inconsistent with one 
Fig. 3 Bilateral networks for the financial system shown in Fig. 1
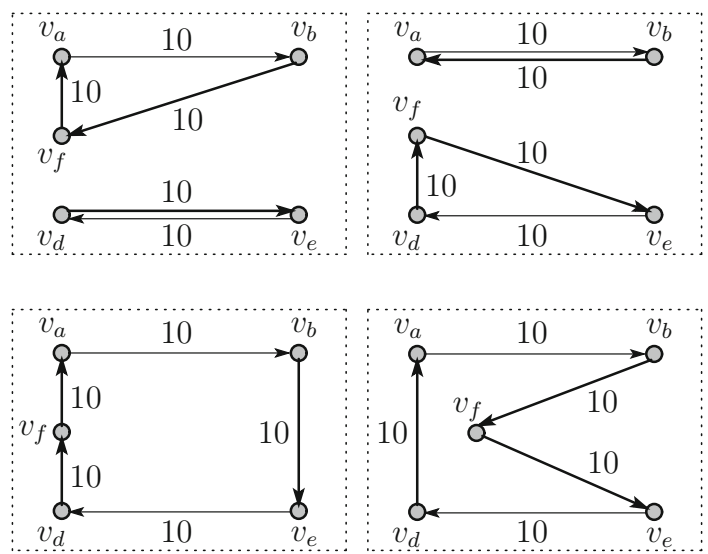

of the assumptions. The left financial system does not satisfy Assumption 1, the middle financial system does not satisfy Assumption 2, and the right financial system does not satisfy Assumption 3.

\subsection{Stage 1: CCP scheme/bilateral scheme}

At Stage 1, the hypothetical entity is materialized under either of the two schemes: the CCP scheme and the bilateral scheme. For the CCP scheme, the hypothetical entity is itself reinterpreted as a CCP for the target security. Thus, the network given at Stage 0 is unchanged. When the given network as Stage 0 is that shown in Fig. 1 with hypothetical entity $v_{c}, v_{c}$ is reinterpreted simply as the CCP under the CCP scheme at Stage 1. Note that the CCP does not offset obligations at Stage 1, but it will do so at Stage 2. In order to simplify relevant statements, we refer to a network derived under the CCP scheme at Stage 1 as a CCP network.

For the bilateral scheme, the hypothetical entity is made to vanish, and we examine all the possible bilateral trades for the target security. We refer to networks that are derived under the bilateral scheme as bilateral networks. Specifically, when the financial system shown in Fig. 1 is given at Stage 0 with hypothetical entity $v_{c}$, we derive four bilateral networks, as shown in Fig. 3. Observe that in each bilateral network, each obligation $\left(v, v_{c}\right), v \in\left\{v_{b}, v_{d}, v_{f}\right\}$ that is previously owed to the hypothetical entity is paired with an obligation $\left(v_{c}, v^{\prime}\right), v^{\prime} \in\left\{v_{a}, v_{e}, v_{f}\right\}$ that is previously owed by the hypothetical entity, and the pair of arcs is replaced with a new arc $\left(v, v^{\prime}\right)$. For the network shown on the upper-left in Fig. 3, the relevant arcs are paired such that $\left\{\left(v_{b}, v_{c}\right),\left(v_{c}, v_{f}\right)\right\},\left\{\left(v_{f}, v_{c}\right),\left(v_{c}, v_{a}\right)\right\},\left\{\left(v_{d}, v_{c}\right),\left(v_{c}, v_{e}\right)\right\}$, and each of the pairs is replaced by each of $\left(v_{b}, v_{f}\right),\left(v_{f}, v_{a}\right)$, and $\left(v_{d}, v_{e}\right)$.

In general, for a given financial system $\left(\mathcal{N}=\langle V, A, f\rangle, v^{*} \in V\right)$, let $A_{t o} \subset A$ denote a set of obligations owed to the hypothetical entity, and $A_{b y} \subset A$ denote a set of obligations owed by the hypothetical entity. Note that $\left|A_{t o}\right|=\left|A_{b y}\right|{ }^{7}$ Consider

\footnotetext{
${ }_{7}$ For a network $\mathcal{N}$ shown in Fig. 1 with hypothetical entity $v_{c}, A_{t o}=\left\{\left(v_{b}, v_{c}\right),\left(v_{d}, v_{c}\right),\left(v_{f}, v_{c}\right)\right\}$, and $A_{b y}=\left\{\left(v_{c}, v_{a}\right),\left(v_{c}, v_{e}\right),\left(v_{c}, v_{f}\right)\right\}$, with $\left|A_{t o}\right|=\left|A_{b y}\right|=3$.
} 

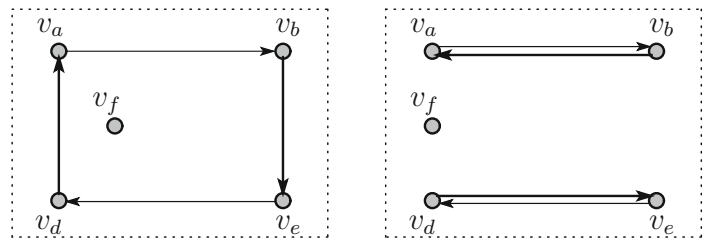

Fig. 4 Networks that are not assumed as bilateral networks for the financial system shown in Fig. 1. For the network shown Fig. 1, each of the networks shown in the figures is derived when the pair of arcs $\left\{\left(v_{c}, v_{f}\right),\left(v_{f}, v_{c}\right)\right\}$ is replaced with the vertex $v_{f}$. We do not assume these networks as relevant bilateral networks since it effectively assumes offsetting of obligations under the bilateral scheme.

Fig. 5 The net-out CCP network for the financial system shown in Fig. 1. The pair of arcs $\left\{\left(v_{f}, v_{c}\right),\left(v_{c}, v_{f}\right)\right\}$ has been removed from the network shown in Fig. 1

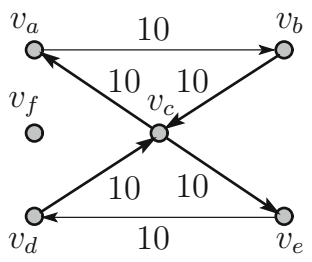

all the possible one-to-one matchings between $A_{t o}$ and $A_{b y}$. For each matching, let each pair of $\operatorname{arcs}\left\{\left(v, v^{*}\right),\left(v^{*}, v^{\prime}\right)\right\}, v, v^{\prime} \in V$ be replaced with a new arc $\left(v, v^{\prime}\right)$. Here, we exclude any matching that includes a pair of arcs regarding the same vertex such that $\left\{\left(v, v^{*}\right),\left(v^{*}, v\right)\right\}, v \in V .^{8}$ The reason for the exclusion of such matching is that the pair of obligations $\left\{\left(v, v^{*}\right),\left(v^{*}, v\right)\right\}$ needs be replaced with vertex $v$, which effectively assumes offsetting between the paired obligations. We assume any bilateral or multilateral offsetting is not executed under the bilateral scheme.

In examining bilateral networks, our later analysis examines all the possible matchings instead of focusing on any specific probable matching. In this sense, we do not make any assumption about counterparty risks conceived among financial institutions, or other factors that affect probable realization of matching.

\subsection{Stage 2: Offsetting under the CCP scheme}

Stage 2 is relevant only to networks derived under the CCP scheme. The CCP now offsets obligations, and we derive an associated network. For example, when we see the network in Fig. 1 as a CCP network with CCP $v_{c}$, offsetting by the CCP derives a network shown in Fig. 5, where the pair of obligations $\left\{\left(v_{f}, v_{c}\right),\left(v_{c}, v_{f}\right)\right\}$ has been offset. In general, for a CCP network $\mathcal{N}=\langle V, A, f\rangle$ with CCP $v^{*} \in V$, remove all the arcs that constitute a pair $\left\{\left(v, v^{*}\right),\left(v^{*}, v\right)\right\}$, for $v \in V$. We call the derived network the net-out CCP network regarding $v^{*}$. Our aim is to compare the set of bilateral networks and the net-out CCP network, given the same financial system. When the given finan-

\footnotetext{
${ }^{8}$ For network $\mathcal{N}$ shown in Fig. 1 with hypothetical entity $v_{c}$, we exclude one-to-one matchings in which the pair of $\operatorname{arcs}\left\{\left(v_{f}, v_{c}\right),\left(v_{c}, v_{f}\right)\right\}$ is included. Those excluded matchings would yield networks shown in Fig. 4.
} 

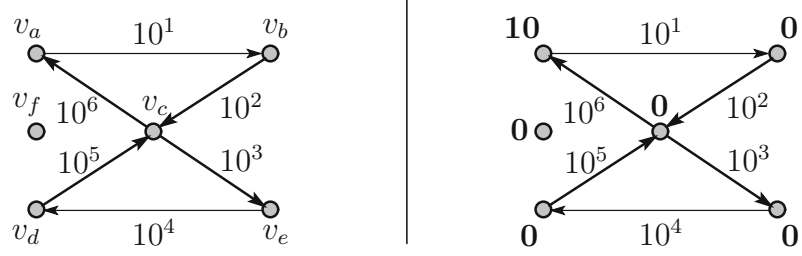

Fig. 6 Example sequence and relevant liquidity needs (1). $s\left(v_{a}, v_{b}\right)=1, s\left(v_{b}, v_{c}\right)=2, s\left(v_{c}, v_{e}\right)=3$, $s\left(v_{e}, v_{d}\right)=4, s\left(v_{d}, v_{c}\right)=5, s\left(v_{c}, v_{a}\right)=1, p_{v_{a}}(s)=10$, and $p_{v_{b}}(s)=p_{v_{c}}(s)=p_{v_{d}}(s)=p_{v_{e}}(s)=$ $p_{v_{f}}(s)=0$

cial system is a network shown in Fig. 1 with hypothetical entity $v_{c}$, we compare the set of bilateral networks shown in Fig. 3 with the net-out CCP network shown in Fig. 5.

\subsection{Stage 3: Settlement of obligations}

At Stage 3, the remaining obligations are settled on a gross basis. ${ }^{9}$ Specifically, we define a gross settlement for a network $\mathcal{N}=\langle V, A, f\rangle$ with one-to-one mapping (sequence) $s: A \rightarrow\{1,2, \ldots,|A| \mid\}$ and a set of values $\left\{p_{v}(s) \geq 0\right\}_{v \in V}$, in which the former shows the relative order of the settlements, while the latter shows the liquidity needs for each $v \in V$ under the order. For a network shown in Fig. 5, an example sequence $s: A \rightarrow\{1,2, \ldots, 6\}$ is shown on the left side of Fig. 6, in which $s(a)$ is written on the upper-right of the value $f(a)$ for each $a \in A$. The right side of the figure shows the same network to which is added the corresponding $p_{v}(s)$ for each $v \in V$, where each value is shown in boldface. Under the specified order, the financial institution expressed as $v_{a}$ needs to input 10 funds, since it has not received any liquidity before. For each of the rest of the institutions, including the $\mathrm{CCP}$, the liquidity needs are shown as zero, since each has received sufficient amount of liquidity when each settles its obligation. Thus, we set $\left\{p_{v}(s)\right\}_{v \in V}$ so that $p_{v}(s)$ is neither redundant nor short of the settlements for each $v \in V$ under given sequence $s$. Formally, we explicitly set $\left\{p_{v}(s)\right\}_{v \in V}$ in the following procedure.

- Procedure to set $\left\{p_{v}(s)\right\}_{v \in V}$

.Let $\langle V, A, f\rangle$ and $s: A \rightarrow\{1,2, . .,|A|\}$ be given.

. Let $k=0,1,2, \ldots$, show the current order for the relevant settlements.

. Let $p_{v}^{h}(k)$ indicate the current liquidity holding.

- Let $p_{v}^{d}(k)$ indicate the current liquidity needs.

\section{- Initialization}

- Set $p_{v}^{h}(0)=0$ and $p_{v}^{d}(0)=0$, for every $v \in V$.

- Set $k=1$.

\footnotetext{
9 The World Bank (2013) reports that more than $80 \%$ of the surveyed payment systems had adopted real-time gross settlement (RTGS) systems. Several interbank payment systems incorporate offsetting mechanisms into their RTGS systems, which are referred to as the liquidity saving mechanism. From this perspective, this study assumes that settlements at Stage 3 are under an RTGS system without any liquidity saving mechanism.
} 


\section{- Main Procedure}

Take $a=\left(v, v^{\prime}\right) \in A$ such that $s(a)=k$.

i) For the payer $v$, set $p_{v}^{d}(k)$ and $p_{v}^{h}(k)$ as follows.

$$
\begin{aligned}
& \cdot p_{v}^{d}(k)=\max \left(f(a)-p_{v}^{h}(k-1), 0\right), \\
& \cdot p_{v}^{h}(k)=\max \left(p_{v}^{h}(k-1)-f(a), 0\right) .
\end{aligned}
$$

ii) For the receiver $v^{\prime}$, set $p_{v^{\prime}}^{d}(k)$ and $p_{v^{\prime}}^{h}(k)$ as follows.

$$
\begin{aligned}
& \cdot p_{v^{\prime}}^{d}(k)=0, \\
& \cdot p_{v^{\prime}}^{h}(k)=p_{v^{\prime}}^{h}(k-1)+f(a) .
\end{aligned}
$$

iii) For the other $v^{\prime \prime} \in V \backslash\left\{v, v^{\prime}\right\}$, set $p_{v^{\prime \prime}}^{d}(k)=0$ and $p_{v^{\prime \prime}}^{h}(k)=p_{v^{\prime \prime}}^{h}(k-1)$.

- Update the current order as $k:=k+1$.

- If $k>|A|$, proceed to the finalization; otherwise, repeat the main procedure with the updated $k$.

\section{- Finalization}

$$
\text { - For each } v \in V \text {, set } p_{v}(s)=\sum_{k=1}^{|A|} p_{v}^{d}(k) \text {. }
$$

For given network $\langle V, A, f\rangle$ and sequence $s: A \rightarrow\{1,2, \ldots,|A|\}$, we examine total liquidity needs $\sum_{v \in V} p_{v}(s)$ for our analysis. Note that we refer to liquidity needs as the amount of liquidity necessary for the relevant settlements, but not the amount of liquidity that is prepared by financial institutions from an ex-ante perspective. The purpose is to clarify liquidity needs in relation to the overall financial state, independent of expectations held by the relevant financial institutions that could depend on each individual context. The overall financial state is expressed with each of our liquidity scenarios.

\subsubsection{Liquidity scenarios}

We focus on two polar types of scenario for our analysis; one is a scenario in the good environment, and the other in the bad environment. The good environment refers to the financial state in which settlements are executed under the best coordination, and the overall liquidity needs is the minimum possible. By contrast, the bad environment refers to the state in which settlements are executed under the worst coordination, and liquidity needs are the maximum possible. The bad environment is meant to express liquidity needs during times of financial distress, while the good environment is used as a benchmark state in times of non-distress. Given a net-out CCP network in Fig. 5, Fig. 6 shows a settlement in the good environment, and Fig. 7 shows a settlement in the bad environment for the same network.

Formally, the total liquidity needs in each environment is derived by each of the following minimization and maximization problems.

\section{(Liquidity problem for the good environment) ${ }^{10}$}

Given network $\langle V, A, f\rangle$, take one-to-one mapping $s: A \rightarrow\{1,2, \ldots,|A|\}$ and associated $\left\{p_{v}(s)\right\}_{v \in V}$ such that

$$
\min _{s} \sum_{v \in V} p_{v}(s) \text {. }
$$

\footnotetext{
10 The presented minimization and maximization problems are formally introduced in Hayakawa (2016), Hayakawa (2014), and originally in his doctoral thesis. In arguing the computational aspect, the problems are specifically referred to as the (minimum/maximum) settlement fund circulation problem.
} 

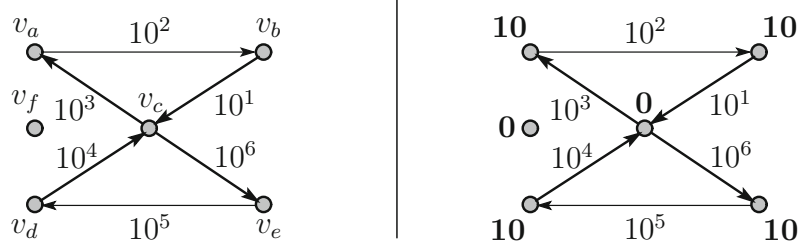

Fig. 7 Example sequence and relevant liquidity needs (2) $s\left(v_{a}, v_{b}\right)=1, s\left(v_{b}, v_{c}\right)=2, s\left(v_{c}, v_{e}\right)=3$, $s\left(v_{e}, v_{d}\right)=4, s\left(v_{d}, v_{c}\right)=5, s\left(v_{c}, v_{a}\right)=1, p_{v_{a}}(s)=10$, and $p_{v_{b}}(s)=p_{v_{c}}(s)=p_{v_{d}}(s)=p_{v_{e}}(s)=$ $p_{v_{f}}(s)=0$

Fig. 8 Settlements in the good environment
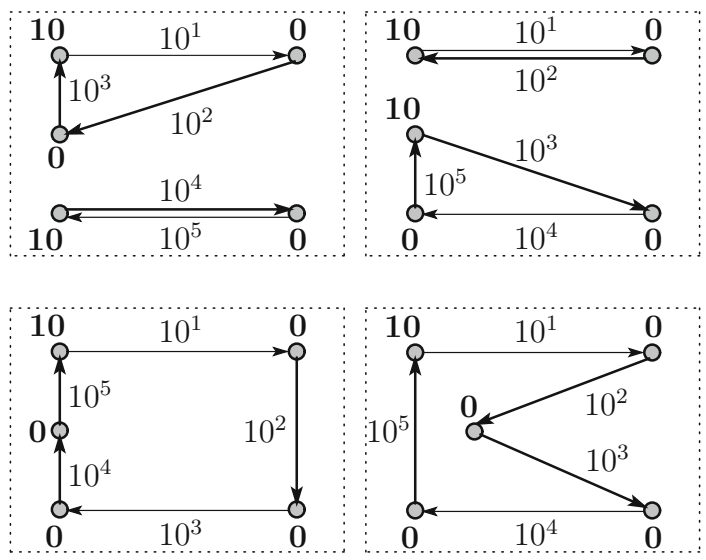

\section{(Liquidity problem for the bad environment)}

Given network $\langle V, A, f\rangle$, take one-to-one mapping $s: A \rightarrow\{1,2, \ldots,|A|\}$ and associated $\left\{p_{v}(s)\right\}_{v \in V}$ such that

$\max _{s} \sum_{v \in V} p_{v}(s)$.

In other words, the liquidity problem for the good (bad) environment derives the minimum (maximum) total liquidity needs with respect to every possible order of settlements. For clarity of the problems, take the networks shown in Fig. 3 as inputs for each of the minimization and maximization problems. Then, Fig. 8 shows relevant settlements in the good environment, and Fig. 9 shows those in the bad environment.

The bad environment could be interpreted as a panic situation that is typical in times of financial distress, whereby financial institutions try to receive their payments as early as possible, or require margin for their relevant exposure. Notice that in our model, we do not allow multiple settlements to be made simultaneously, since we assume that each different order needs to be set for each arc. That means not all the obligations are settled with additional liquidity input, as confirmed in Fig. 7, where $p_{v_{c}}=0$ instead of 20. In other words, a certain positive amount of liquidity is economized even under the bad environment. This is because when the first payment is made, there is at least one institution that receives liquidity before it makes any payment. This captures the essential nature of the circulation of liquidity such that liquidity can be used once it is obtained. The good environment is treated as an ideally efficient situation in terms of 
Fig. 9 Settlements in the bad environment
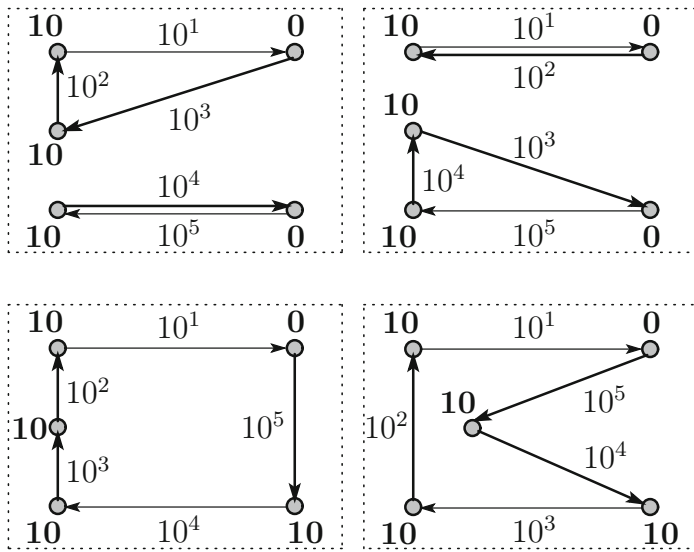

liquidity input, in which the liquidity inputs, and associated costs, are economized as much as possible.

\section{Illustrative examples}

We argue that the effect of a CCP needs be examined carefully in each liquidity scenario. We show that in the bad environment, a CCP increases overall liquidity needs if the netting efficiency is not sufficiently high. Still, higher netting efficiency contributes to decrease liquidity needs in the bad environment, and sufficiently high netting efficiency ensures that the CCP decreases the total liquidity needs compared to the corresponding bilateral settlements. However, when we consider the good environment, higher netting efficiency does not necessarily serve to decrease overall liquidity needs. Actually, it is possible that higher netting efficiency leads to larger overall liquidity needs. Thus, there is a possible trade-off regarding the netting service provided by the CCP.

In order to illustrate the trade-off and our relevant analysis in a less formal manner, here, we examine three example financial systems (A), (B), and (C), shown in Fig. 10. Hypothetical entity is $v_{c}$ in each financial system. As easily confirmed, the amount of obligations offset by the CCP is the largest for (C), and zero for (A).

Figure 11 shows settlements for networks generated by financial system (A). Although we have more than one bilateral network, for illustrative purposes, we focus on one bilateral network shown in the figure. In the analysis section, we show results considering all possible bilateral networks for each given financial system. Figure 11 shows an example order of settlements and the relevant liquidity needs for each of the two networks (a net-out CCP network and a bilateral network) in each environment. Observe that in the bad environment, the total liquidity needs are larger for the CCP scheme, while it is the same in the good environment. In the same manner, Fig. 12 shows relevant settlements for financial system (B), and Fig. 13 shows those for financial system (C). Notice that the offsetting amount increases when we move from (A) to $(\mathrm{B})$ and from (B) to (C). Thus, we observe that as the offsetting amount increases, 
(A)

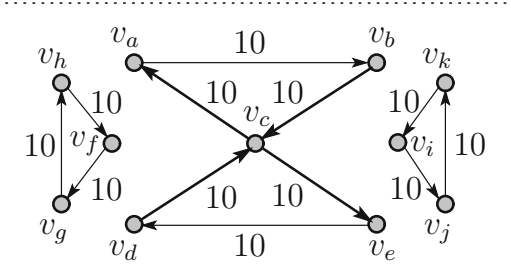

(B)

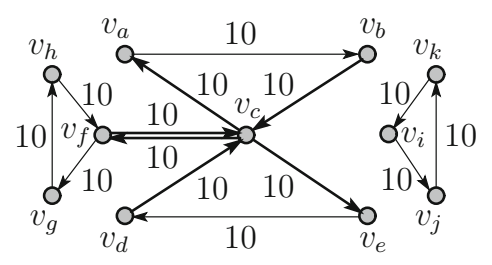

(C)

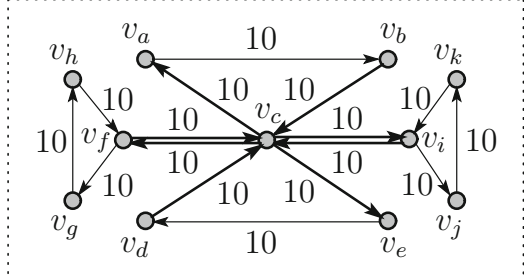

Fig. 10 Example financial systems. In each financial system (A), (B), and (C), $v_{c}$ is assumed as the hypothetical entity

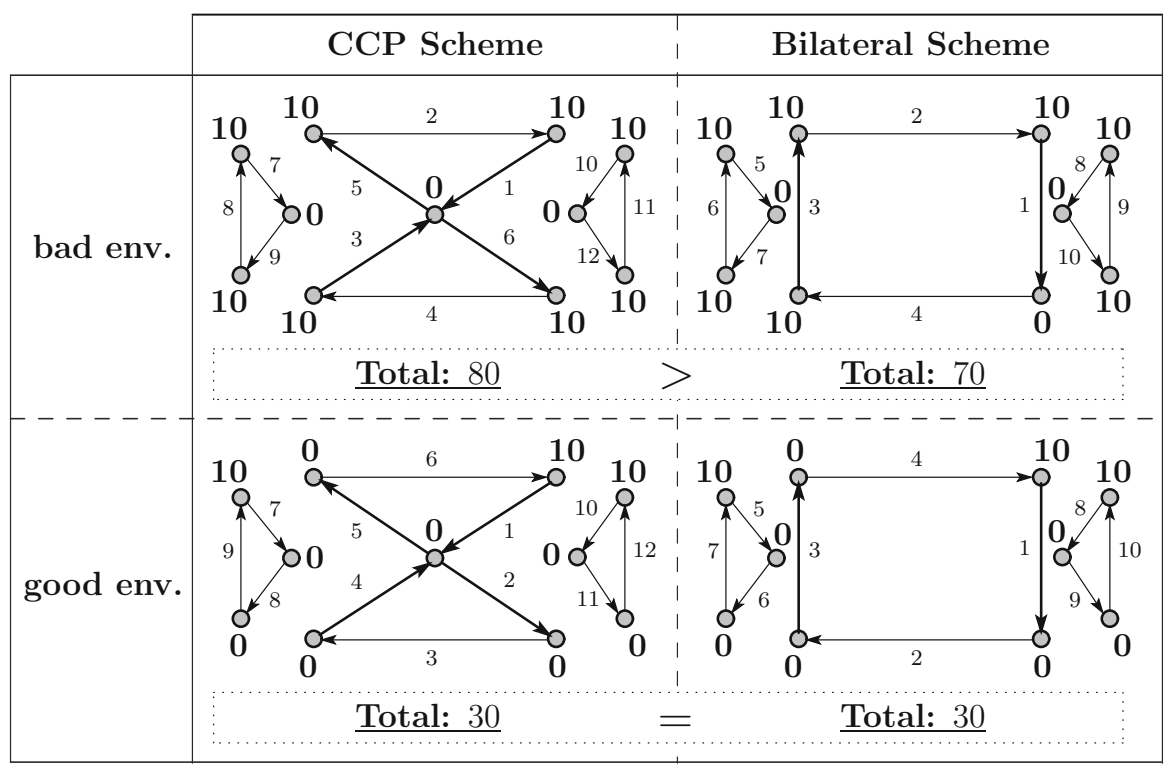

Fig. 11 Financial system (A): relevant settlements. For each network, relevant order is presented for each arc and the corresponding liquidity needs are presented for each vertex. We omit the relevant amount of obligations, which is 10 for all the arcs, in this figure

the total liquidity needs under the CCP scheme become relatively smaller in the bad environment, but relatively larger in the good environment. This shows the possible trade-off of the multilateral offsetting by the CCP. 


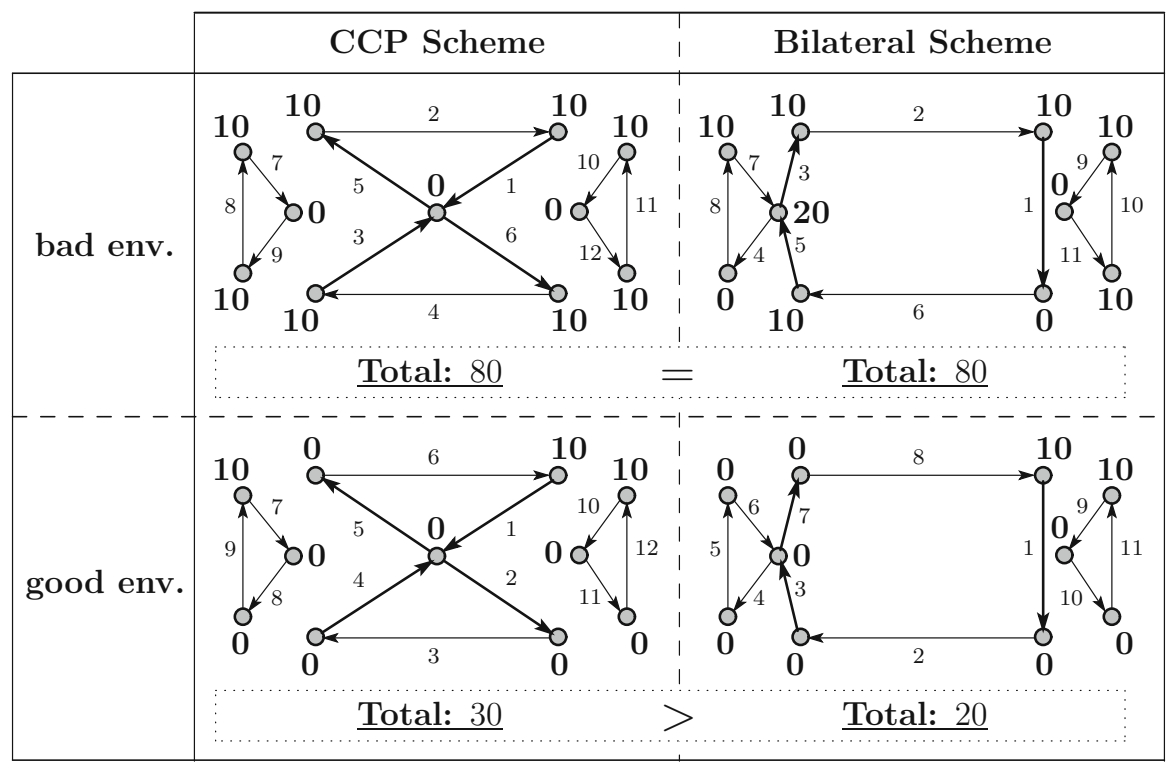

Fig. 12 Financial system (B): relevant settlements

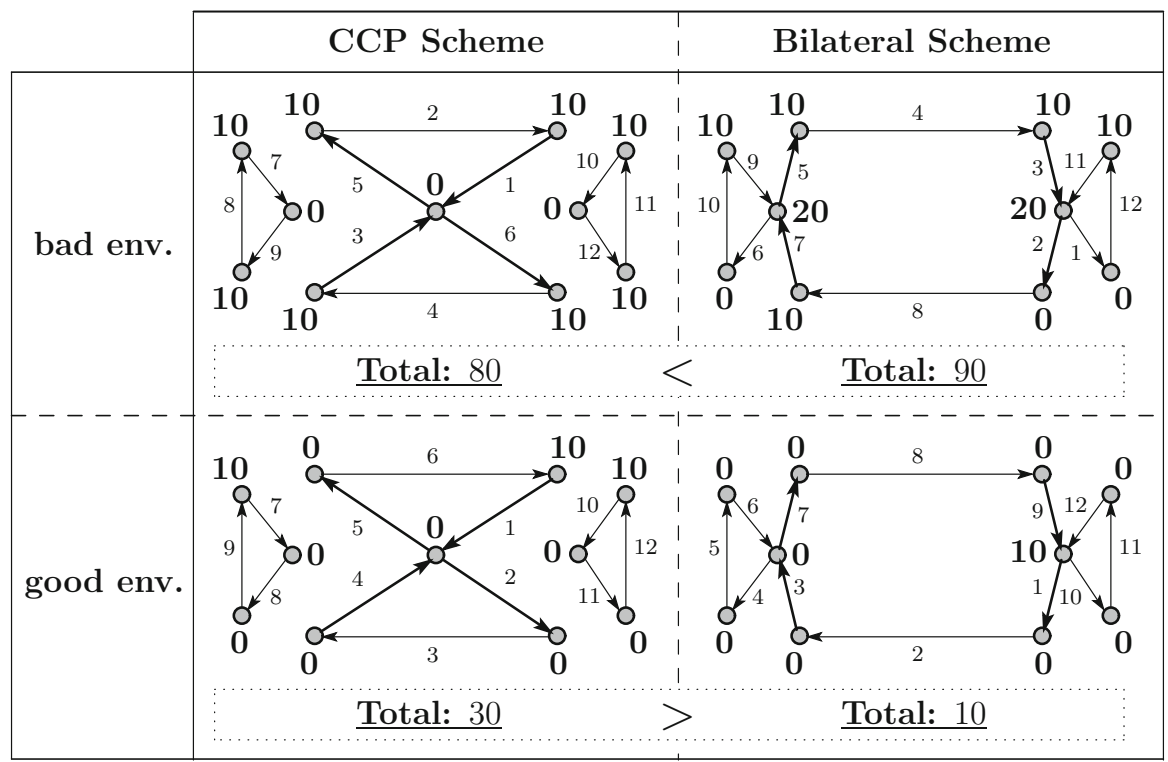

Fig. 13 Financial system (C): relevant settlements

The trade-off of the multilateral offsetting by the CCP is better understood by decomposing the total effect of the CCP into two types. One type is referred to as the central routing effect, and the other as the central netting effect. The base result shown in the analysis section is summarized as follows. 


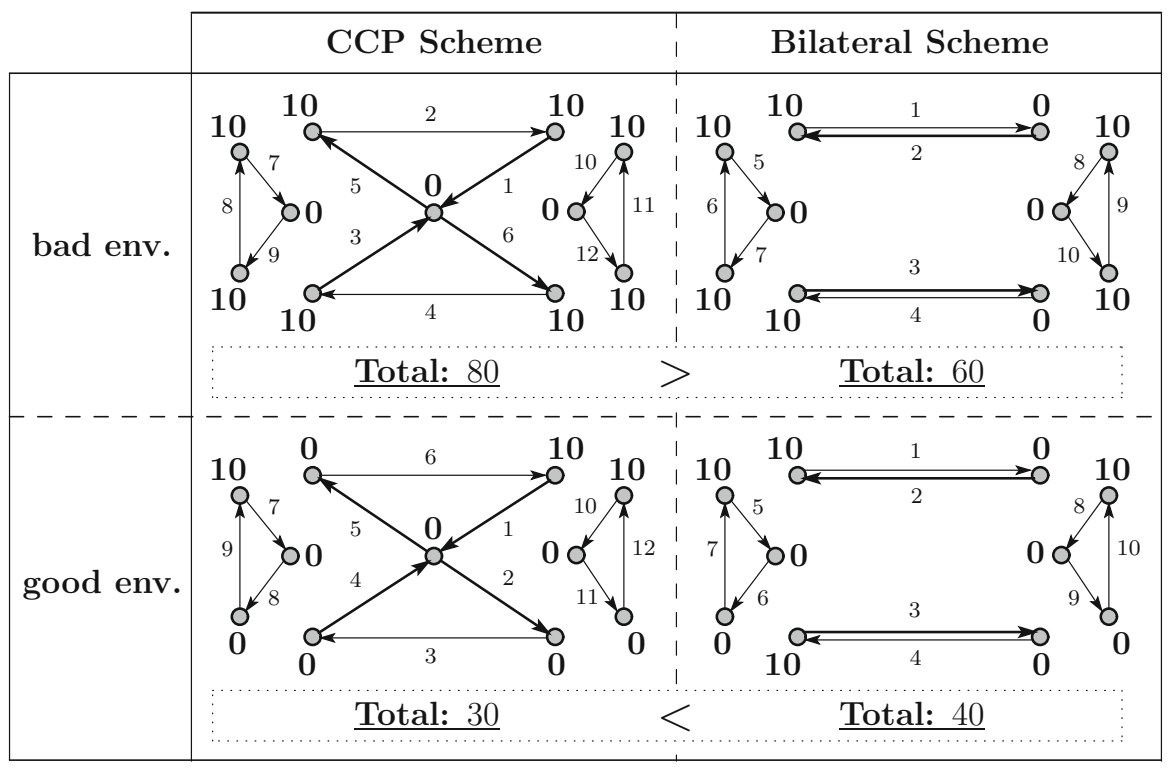

Fig. 14 Financial system (A): relevant settlements (2)

$$
(\text { Total effect })=(\text { Central netting effect })+(\text { Central routing effect }) .
$$

The base result essentially states that the total effect is quantitatively decomposed into the two effects, such that the two effects are additive with each other.

\subsection{Central routing effect}

For financial system (A), there is no offsetting under the CCP scheme, which lets the net-out CCP network be equal to the CCP network. Thus, the total effect of the CCP for financial system (A) equals the central routing effect. Furthermore, the central routing effect of the CCP for each financial system (B) and (C) is equal to that for financial system (A), from the observation that each net-out CCP network for financial system (B) and (C) is essentially the same as the CCP network for financial system (A). This is formally shown in the analysis section.

For financial system (A) and relevant networks, as shown in Fig. 11, the liquidity needs in the bad environment are larger for the net-out CCP network than the shown bilateral network. This also holds when we compare the net-out CCP network to another bilateral network, as shown in Fig. 14. In general, we show that the central routing effect in the bad environment is strictly negative. By comparison, as indicated by the two figures, the liquidity needs for the net-out CCP network in the good environment is either equal to or smaller than that for each of the relevant bilateral networks. In general, we show that the central routing effect in the good environment is weakly positive in that sense.

The intuition for the results presented above is as follows. In the good environment, the CCP tends to provide additional routes for liquidity to circulate more efficiently. 
This is well illustrated in Fig. 14. For the bilateral network shown on the right of the figure, there are four mutually isolated cycles of obligations. When we turn to the net-out CCP network shown on the left, we observe that the CCP serves to connect two of the previous isolated cycles to let them form one cycle. The same liquidity can now circulate throughout the united cycle. By contrast, in the bad environment, the CCP provides an additional stop for liquidity, which always increases the total liquidity needs. This is easier to observe in Fig. 11. Each of the bilateral and net-out CCP networks consists of three mutually isolated cycles. The difference is that for the net-out CCP network, there is one additional vertex for one of the cycles (the cycle at the center). This actually increases the liquidity needs for the relevant cycle from 30 to 40 .

The central routing effect clarifies a negative aspect of adding a CCP during times of financial distress. For the case of derivative contracts, the CCP tends to demand liquidity in the form of margin. Suppose that the relevant derivatives are traded without any CCP; then, it is possible that the direct counterparty instead of the CCP demands margin, intending to ensure counterparty risk. Suppose that for some derivative trade, the margin demanded by the CCP is the same level as that demanded by the direct counterparty; then, there is no change in the amount of the required margin for the trade itself. However, during times of financial distress when margin requirement is prevalent regardless of the types of derivatives, adding a CCP indicates that the number of institutions that require margin effectively increases in total. This is interpreted as a negative externality of adding a CCP, which is well demonstrated by the central routing effect.

\subsection{Central netting effect}

In order to observe the central netting effect for a given financial system, we take a corresponding financial network as the standard for comparison. Specifically, for each financial system (B) and (C) shown in Fig. 10, the standard financial system is financial system (A). In general, for a given financial system, we take the standard financial system by offsetting all the obligations regarding the hypothetical entity. For financial systems (B) and (C), the hypothetical entity is $v_{c}$, and offsetting the relevant obligations yields financial system (A). Our view is that the central routing effect for a given financial system is the same as that for its standard financial system, and the central netting effect is captured by comparing the bilateral networks for the two financial systems. For example, a bilateral network for financial system (B) shown on the right of Fig. 15 is compared with a bilateral network for financial system (A) shown on the left of the same figure. The way to correspond the relevant bilateral networks is shown in the analysis section. We observe there are two mutually isolated cycles in the bilateral network for financial system (B), while there are three for (A). ${ }^{11}$ We consider that the original larger cycle in (B) is separated into two cycles (one with three vertices and one with four vertices) in (A). In the bad environment, the separation tends to decrease the number of stops for liquidity, and accordingly

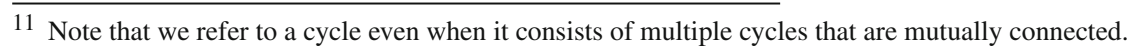




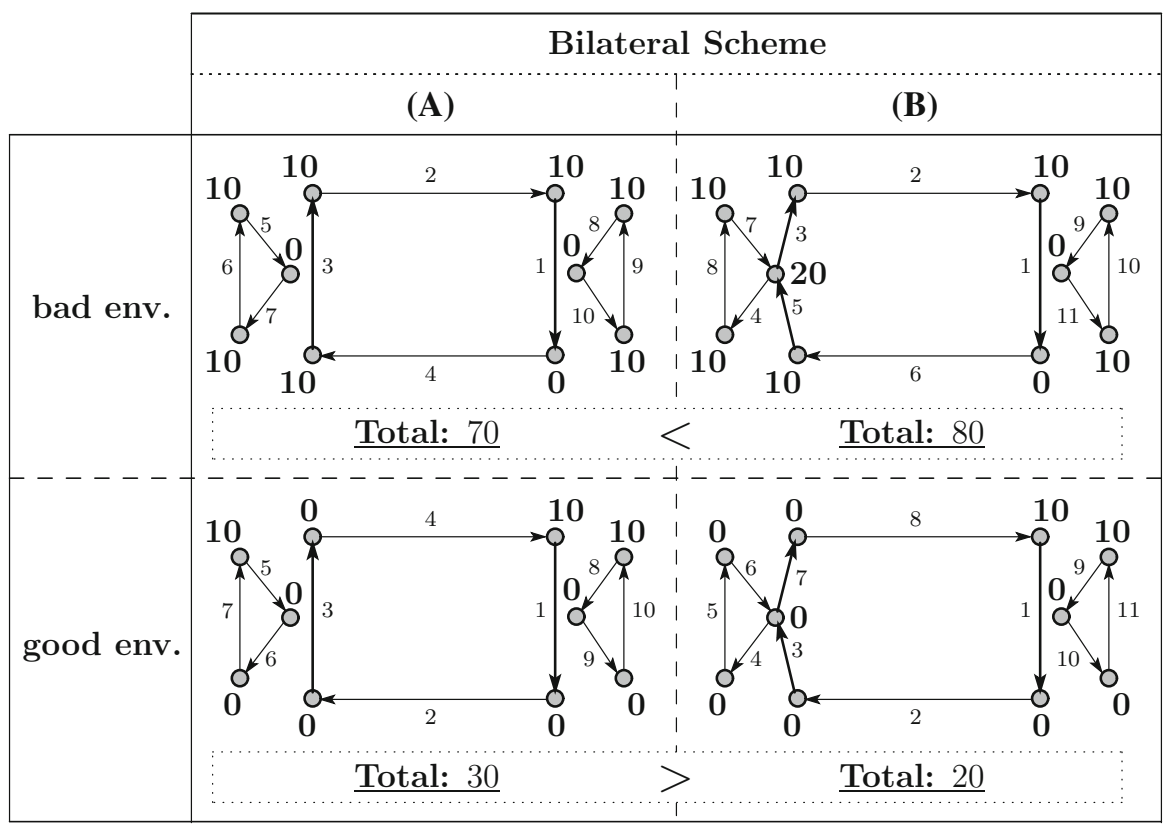

Fig. 15 Financial systems (A) and (B): networks under the bilateral scheme

decreases the liquidity needs. In general, we show that the central netting effect is weakly positive in the bad environment. By contrast, in the good environment, the separation now could increase liquidity needs. This is because the same liquidity can circulate only within each of the separated cycles. Figure 16 compares a bilateral network for financial system (C) shown on the right and that for financial system (A) shown on the left, for which we confirm the central netting effect in the same manner.

Although we observe a negative aspect of the central netting effect in Figs. 15 and 16, it could conversely have a positive effect. We illustrate this point using financial systems (D) and (E), shown in Fig. 17. Note that (D) is the standard financial system for $(E)$. Thus, the central routing effect for $(E)$ is captured through (D). Figure 18 shows example bilateral networks for our examination of the central netting effect. We observe that the central netting effect is positive in the good environment. We observe three mutually separated cycles for (E) and two for (D). The liquidity needs are reduced by reducing one cycle.

Suppose each of isolated vertices $v_{f}$ and $v_{g}$ in (E) forms a cycle with additional vertices and arcs through the trades of the non-target security, as is the case for financial system (C). Then, whether the central netting effect in the good environment is positive or negative depends on the amount of obligations for the non-target security. Actually, we show that it is positive when the amount of obligations for the non-target security is sufficiently small (financial system $(\mathrm{E})$ is understood as an extreme case such that there is no relevant trade of the non-target security.). 


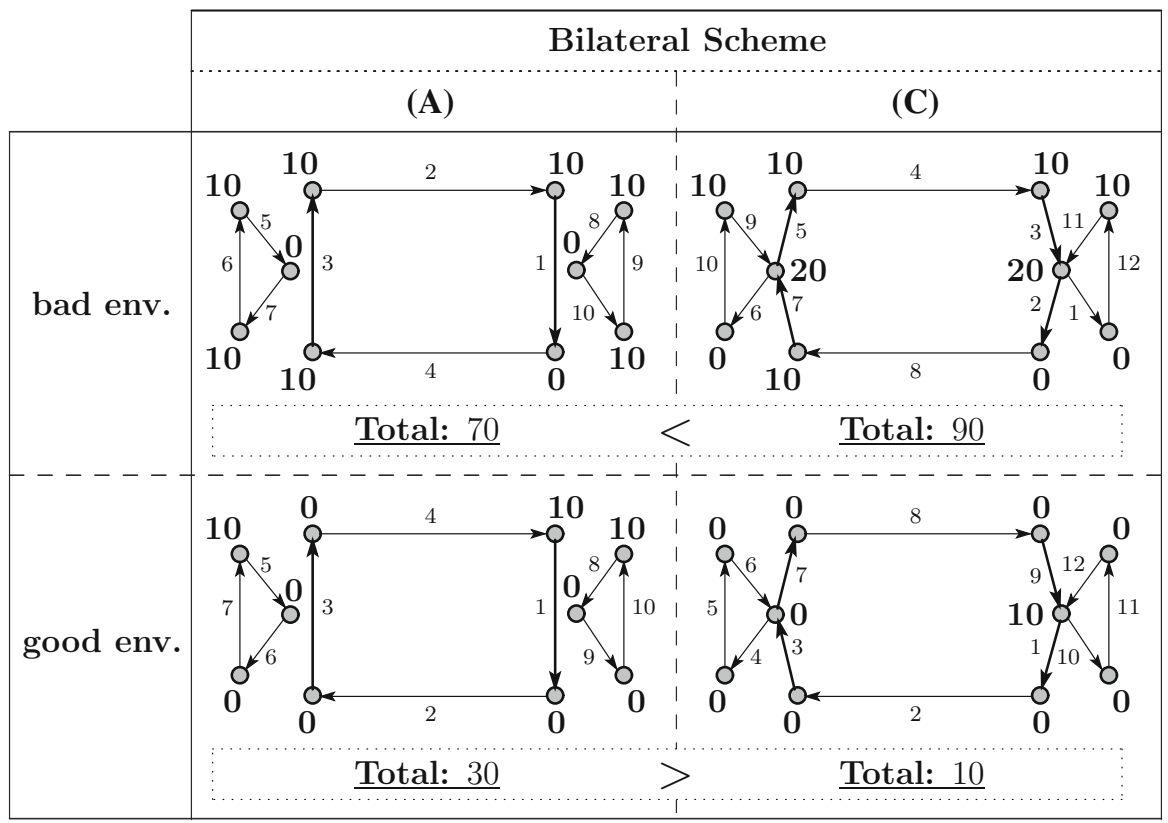

Fig. 16 Financial systems (A) and (C): networks under the bilateral scheme

(D)

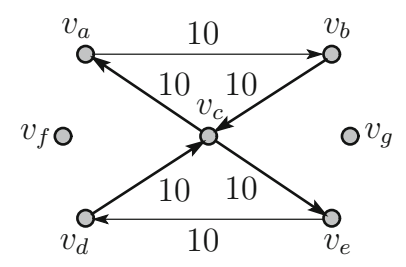

(E)

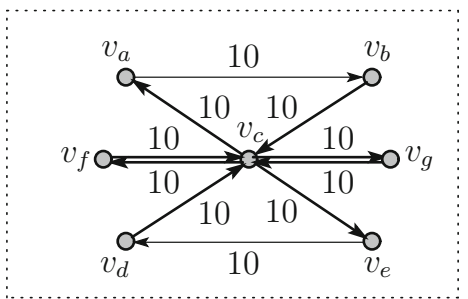

Fig. 17 Financial systems (D) and (E)

On the contrary, we show that the central netting effect is negative in the good environment if the amount of each obligation for the target security is relatively small compared to that for the non-target security. This has a political implication in that the operating cost of CCPs in terms of the overall liquidity needs should not be evaluated solely from the activities of the CCPs themselves, but their externalities on the efficiency of liquidity circulation should be considered with sufficient care.

\section{Overview of the results}

We briefly overview the analysis and relevant results presented in the next section. In the analysis, first, the decomposition of the total effect of adding a CCP is formally 


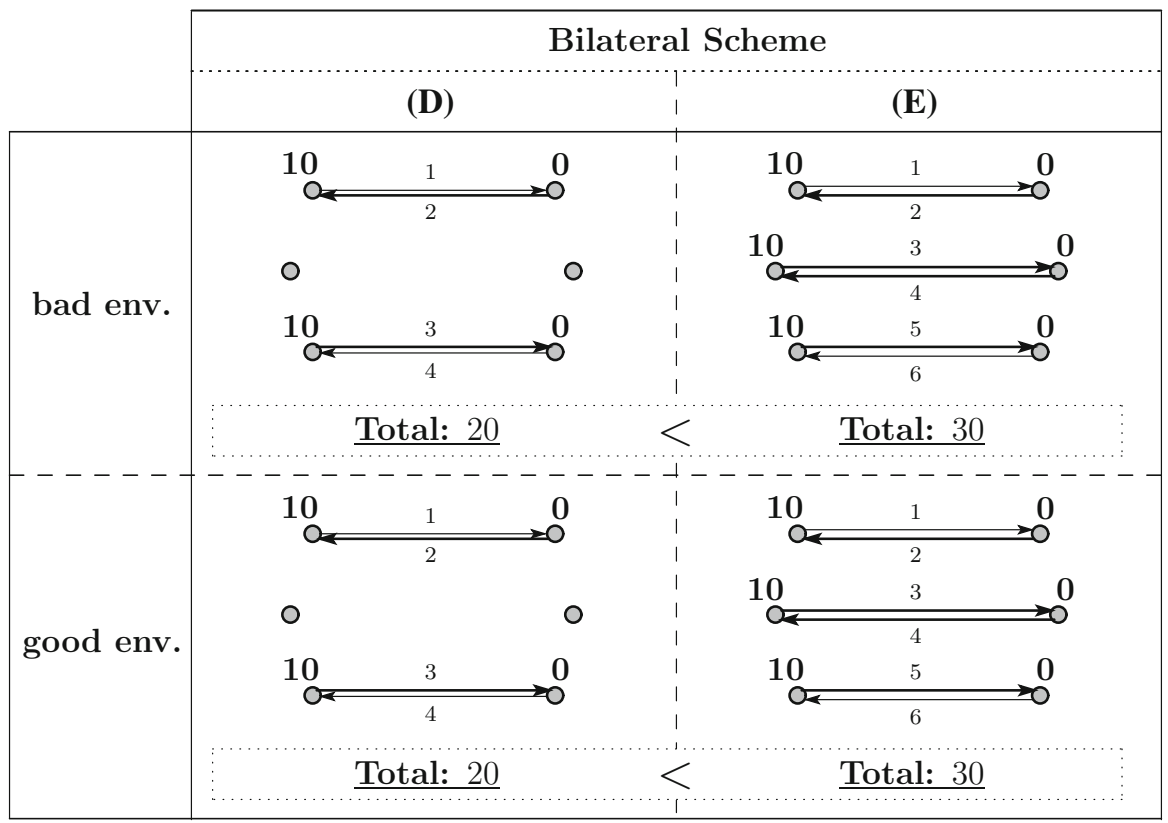

Fig. 18 Financial systems (D) and (E): networks under the bilateral scheme

presented. Although we illustrate the decomposition in Sect. 3 using example bilateral networks for given financial system, Theorem 1 ensures that the decomposition is well defined in the sense that the decomposition is applied to all the possible bilateral networks consistently. The decomposition serves as the analytical basis for showing our relevant results.

Theorem 2 shows the general results for the central routing effect both in the good and bad environment, and Theorem 4 shows the general results for the central netting effect in the bad environment. When we focus on the results in the bad environment, the combination of Theorems 2 and 4 implies that in order for a CCP to have a positive total effect during times of financial distress, it needs to provide sufficiently high netting efficiency.

For the quantitative aspect regarding how much netting efficiency is needed for a $\mathrm{CCP}$ to have a positive effect in the bad environment, we introduce a specific class of financial systems to capture the interconnected feature of real world networks of financial obligations. For the specific class, Theorem 3 shows the quantitative aspect of the central routing effect, while Theorem 5 shows the quantitative aspect of the central netting effect. The results for the combined total effect are summarized in Theorem 6. The theorem shows that the required netting efficiency is $66.6 \%$ for the specific class of financial systems, in order to ensure the total effect of a CCP to be positive in the bad environment. The theorem further explicitly shows the trade-off of multilateral netting by a CCP in that higher netting efficiency leads to a larger negative effect in the good environment when each obligation settled by the CCP is relatively small. 


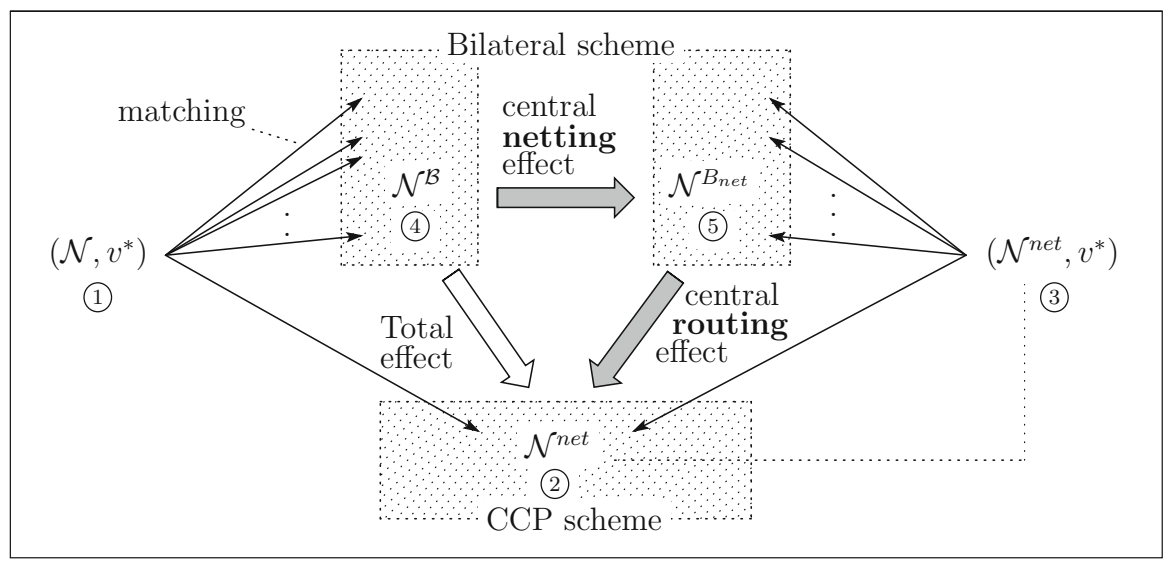

Fig. 19 A schematic illustration of the decomposition of the effect of a CCP. There are two types of arrows shown in the figure: thinner arrows and thicker arrows. Each thinner arrow starts from either of the financial systems, which shows the possible network under each scheme. The three thicker arrows are to illustrate the decomposition

\section{Analysis}

\subsection{Decomposition of the effect of a CCP}

We formally show the decomposition of the effect of a CCP. In order to clarify the statement and relevant notations, Fig. 19 provides a corresponding schematic illustration.

Given financial system $\left(\mathcal{N}, v^{*}\right)(1)$, denote the network derived under the CCP scheme as $\mathcal{N}^{\text {net }}$ (2), which is referred to as the net-out CCP network for $\left(\mathcal{N}, v^{*}\right)$. In an informal description, $\mathcal{N}^{\text {net }}$ is derived from $\left(\mathcal{N}, v^{*}\right)$ by offsetting all obligations regarding $v^{*}$. Then, for the original financial system $\left(\mathcal{N}, v^{*}\right)$, take the corresponding financial system $\left(\mathcal{N}^{n e t}, v^{*}\right)$ (3), which is referred to as a net-out financial system. Note that the net-out CCP network for the obtained net-out financial system $\left(\mathcal{N}^{n e t}, v^{*}\right)$ is the same $\mathcal{N}^{\text {net }}$ (2). ${ }^{12}$

Then, for each bilateral network $\mathcal{N}^{B}$ (4) for the original financial system $\left(\mathcal{N}, v^{*}\right)$ (1)), we take its corresponding bilateral network $\mathcal{N}^{B_{\text {net }}}$ (5) for the obtained financial system $\left(\mathcal{N}^{n e t}, v^{*}\right)$ (3)). The procedure for taking corresponding $\mathcal{N}^{B_{n e t}}$ is specified by (P1) provided below.

For our formal expression regarding the total liquidity needs in each environment, let $x^{\min }(\langle V, A, f\rangle)\left(x^{\max }(\langle V, A, f\rangle)\right)$ denote the total liquidity needs in the good (bad) environment for given network $\langle V, A, f\rangle$. Specifically, $x^{\min }(\langle V, A, f\rangle)=$ $\min _{s} \sum_{v \in V} p_{v}(s)$, and $x^{\max }(\langle V, A, f\rangle)=\min _{s} \sum_{v \in V} p_{v}(s)$, using the notations defined in Sect. 2.4.

The total effect of a CCP in the good environment is examined through a set of values $\left\{x^{\min }\left(\mathcal{N}^{B}\right)-x^{\min }\left(\mathcal{N}^{n e t}\right)\right\}$ with respect to every possible $\mathcal{N}^{B}$, and the effect in the bad environment is examined in exactly the same manner. For now, suppose we have

\footnotetext{
$\overline{12}$ The dotted line in Fig. 19 shows that $\mathcal{N}^{n e t}$ is the same for (2) and (3).
} 
somehow derived a corresponding $\mathcal{N}^{B_{n} \text { et }}$ for each $\mathcal{N}^{B}$. We show our decomposition below, which states that for each $\mathcal{N}^{B}$, the total effect a CCP is decomposed into two effects based on each corresponding $\mathcal{N}^{B_{n e t}}$.

\section{Decomposition of the effect of a CCP}

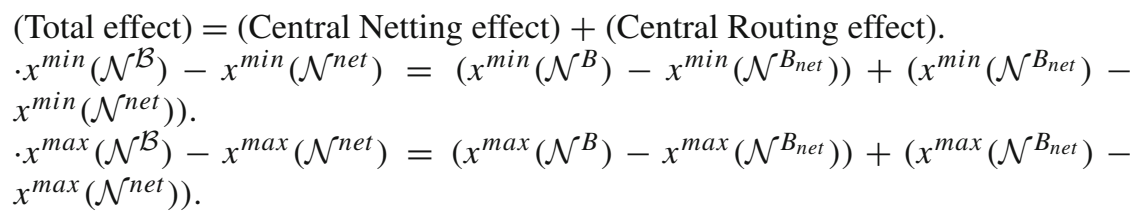

The (P1) procedure below explicitly shows the way to take corresponding $\mathcal{N}^{B_{n e t}}$ for each $\mathcal{N}^{B}$.

\section{1 .1 (P1) procedure and decomposition}

We prepare for the statement of the $(\mathrm{P} 1)$ procedure. For a financial system $(\mathcal{N}=$ $\left.\langle V, A, f\rangle, v^{*} \in V\right)$, take a bilateral network $\mathcal{N}^{B}$. We denote a set of obligations owed to the hypothetical entity as $A_{\text {to }} \subset A$, and a set of obligations owed by the hypothetical entity as $A_{b y} \subset A$. Furthermore, we say $\left\{\left(v, v^{*}\right),\left(v^{*}, v\right)\right\}$ as an offsettable pair with respect to $v$. Let $\mathcal{M}: A_{t o} \rightarrow A_{b y}$ denote a one-to-one matching that yields the bilateral network $\mathcal{N}^{B}$. When $\left\{\left(v, v^{*}\right),\left(v^{*}, v^{\prime}\right)\right\}$ are matched in some matching, then we say $\left(v, v^{\prime}\right)$ is an arc derived by the matching. Given financial system $\left(\mathcal{N}, v^{*}\right)$ and bilateral network $\mathcal{N}^{B}$ derived by one-to-one matching $\mathcal{M}$, the following (P1) procedure yields the corresponding bilateral network $\mathcal{N}^{B_{\text {net }}}$.

\section{(P1) procedure}

- For financial system $\left(\mathcal{N}, v^{*}\right)$, let $f^{m}$ denote the unit price of the target security.

\section{Initialization}

. For $\mathcal{N}^{B_{n e t}}=\left\langle V^{B_{n e t}}, A^{B_{n e t}}, f^{B_{n e t}}\right\rangle$, set $\mathcal{N}^{B_{n e t}}=\mathcal{N}^{B}$.

\section{Main Procedure}

- Take an offsettable pair $\left\{\left(v, v^{*}\right),\left(v^{*}, v\right)\right\}$ for $\left(\mathcal{N}, v^{*}\right)$. Let $\left(v, v^{\prime}\right)$ and $\left(v^{\prime \prime}, v\right)$ be arcs derived by matching $\mathcal{M}$, in which $\left(v, v^{*}\right)$ is matched with $\left(v^{*}, v^{\prime}\right)$, and $\left(v^{*}, v\right)$ is matched with $\left(v^{\prime \prime}, v^{*}\right)$.

1. Remove the pair of $\operatorname{arcs}\left\{\left(v, v^{\prime}\right),\left(v^{\prime \prime}, v\right)\right\}$ from $A^{B_{n e t}}$.

2. Then, if $v^{\prime} \neq v^{\prime \prime}$, add a new $\operatorname{arc}\left(v^{\prime \prime}, v^{\prime}\right)$ to $A^{B_{n e t}}$, and let $f^{B_{n e t}}\left(\left(v^{\prime \prime}, v^{\prime}\right)\right)=f^{m}$.

- Repeat the main procedure until there is no offsettable pair.

Figure 20 explicitly shows how the (P1) procedure works, in which given financial system $\left(\mathcal{N}, v_{c}\right)$ is shown in the upper-right in the box, and the relevant network $\mathcal{N}^{B}$ is shown in the lower-right in the box. The procedure yields $\mathcal{N}^{B_{n e t}}$ shown in the lower-left in the box, through the temporary network shown at the bottom of the figure.

For this specific example, the derived $\mathcal{N}^{B_{n e t}}$ is easily confirmed as a bilateral network for $\left(\mathcal{N}^{n e t}, v_{c}\right)$, where $\mathcal{N}^{n e t}$ is the net-out CCP network for the original financial system $\left(\mathcal{N}, v_{c}\right)$. The first part of the following Theorem 1 ensures that this observation 


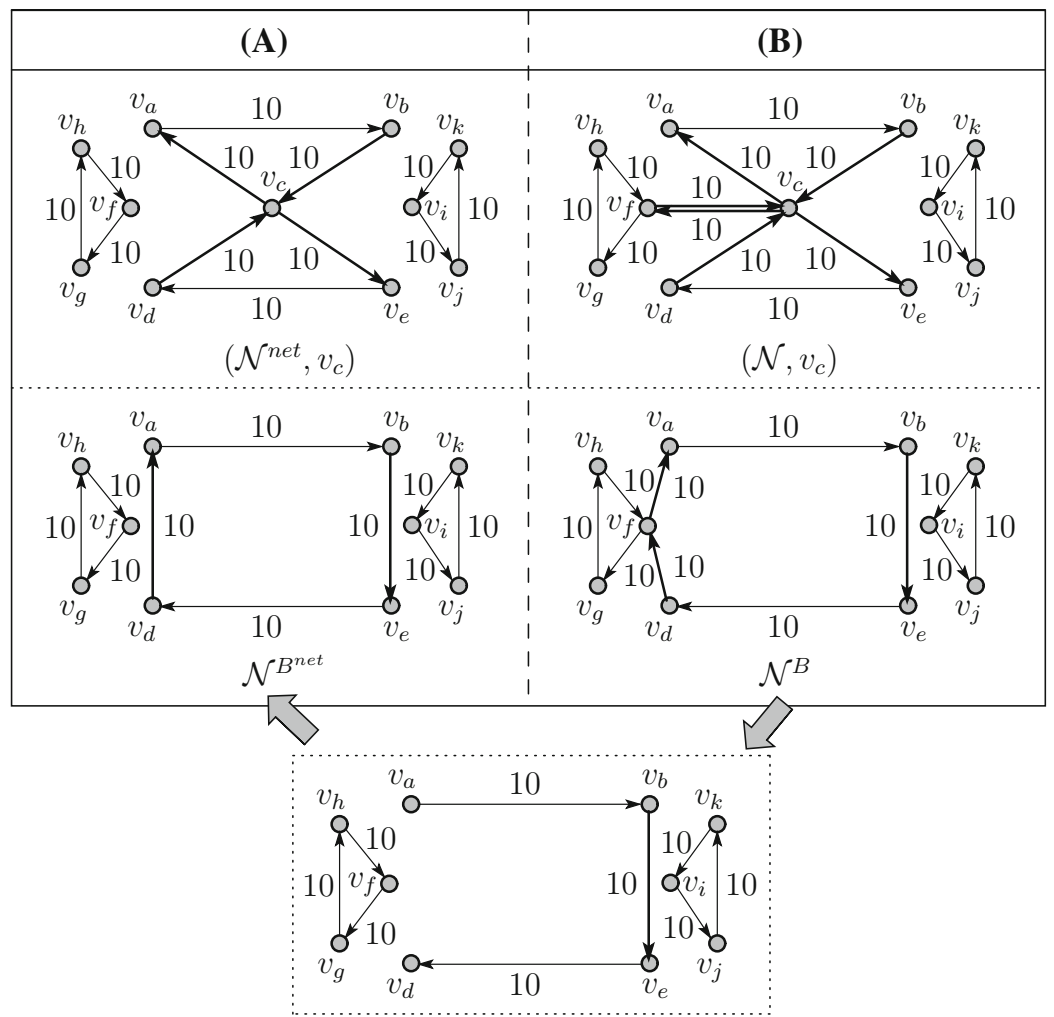

Fig. 20 Example for the (P1) procedure. There is only one offsettable pair for $\left(\mathcal{N}, v_{c}\right)$, which is $\left\{\left(v_{f}, v_{c}\right),\left(v_{c}, v_{f}\right)\right\}$. Under the matching that yields $\mathcal{N}^{B},\left(v_{f}, v_{c}\right)$ is matched with $\left(v_{c}, v_{a}\right)$, while $\left(v_{c}, v_{f}\right)$ is matched with $\left(v_{d}, v_{c}\right)$. Thus, the (P1) procedure removes the corresponding pair of arcs $\left\{\left(v_{d}, v_{f}\right),\left(v_{f}, v_{a}\right)\right\}$, and then, adds a new arc $\left(v_{d}, v_{a}\right)$ with $f\left(\left(v_{d}, v_{a}\right)\right)=10$

holds for each given bilateral network. The second part of the theorem shows consistency of the decomposition for the given financial system. The theorem shows that the decomposition is well-defined.

Theorem 1 Well-defined feature of the decomposition

Given financial system $\left(\mathcal{N}, v^{*}\right)$, denote its net-out CCP network as $\mathcal{N}^{\text {net }}$. We obtain another financial system $\left(\mathcal{N}^{\text {net }}, v^{*}\right)$.

(i) Take arbitrary bilateral network $\mathcal{N}^{B}$ for financial system $\left(\mathcal{N}, v^{*}\right)$. The $(P 1)$ procedure for $\mathcal{N}^{B}$ uniquely yields a bilateral network for financial system $\left(\mathcal{N}^{\text {net }}, v^{*}\right)$.

(ii) For any bilateral network $\mathcal{N}^{B_{n e t}}$ for $\left(\mathcal{N}^{\text {net }}, v^{*}\right)$, there is always a bilateral network $\mathcal{N}^{B}$ for $\left(\mathcal{N}, v^{*}\right)$ such that the $(P 1)$ procedure for $\mathcal{N}^{B}$ yields $\mathcal{N}^{B \text { net }}$.

Proof See Appendix 7.2.

Regarding part (ii) of Theorem 1, observe that for net-out financial system $\left(\mathcal{N}^{\text {net }}, v_{c}\right)$ shown in Fig. 20 , there is another bilateral network. Figure 21 confirms that the $(\mathrm{P} 1)$ procedure actually yields the network, from some different bilateral network $\mathcal{N}^{B}$ for the original financial system $\left(\mathcal{N}, v_{c}\right)$. 


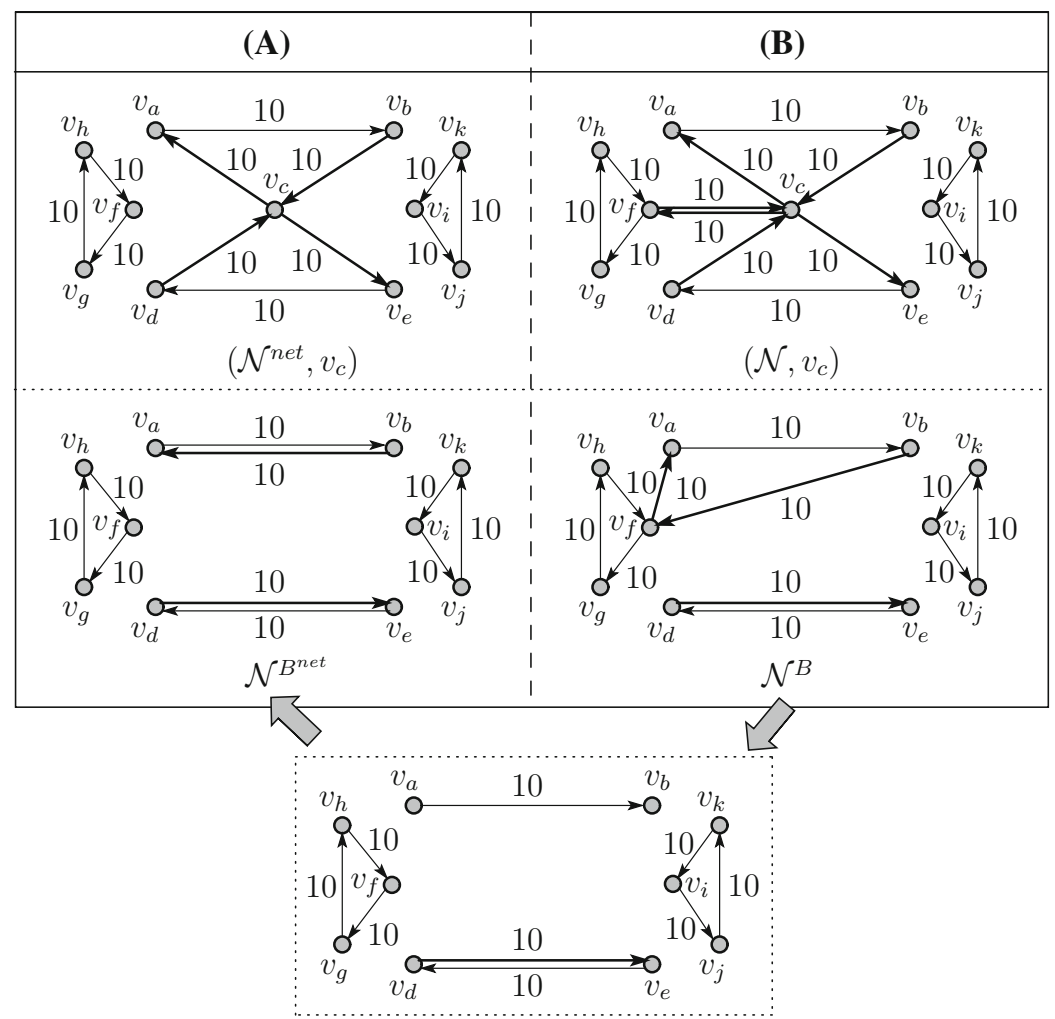

Fig. 21 Another Example for the (P1) procedure. There is only one offsettable pair for $\left(\mathcal{N}, v_{c}\right)$, which is $\left\{\left(v_{f}, v_{c}\right),\left(v_{c}, v_{f}\right)\right\}$. Under the matching that yields $\mathcal{N}^{B},\left(v_{f}, v_{c}\right)$ is matched with $\left(v_{c}, v_{a}\right)$, while $\left(v_{c}, v_{f}\right)$ is now matched with $\left(v_{b}, v_{c}\right)$. Thus, the $(\mathrm{P} 1)$ procedure removes the corresponding pair of arcs $\left\{\left(v_{b}, v_{f}\right),\left(v_{f}, v_{a}\right)\right\}$, and then, adds a new arc $\left(v_{b}, v_{a}\right)$ with $f\left(\left(v_{b}, v_{a}\right)\right)=10$

\subsection{Central routing effect}

\section{Theorem 2 Central routing effect}

(i) The central routing effect is always strictly negative in the bad environment.

(ii) The central routing effect is always weakly positive in the good environment.

Formally, given net-out financial system $\left(\mathcal{N}^{n e t}, v^{*}\right)$, for any bilateral network $\mathcal{N}^{B_{n e t}}$, we obtain

(i) $x^{\max }\left(\mathcal{N}^{B_{\text {net }}}\right)-x^{\max }\left(\mathcal{N}^{\text {net }}\right)<0$.

(ii) $x^{\min }\left(\mathcal{N}^{B_{\text {net }}}\right)-x^{\min }\left(\mathcal{N}^{\text {net }}\right) \geq 0$.

\section{Proof See Appendix 7.3.}

The theorem shows that the central routing effect works in different directions between the good and bad environments. In the good environment, additional CCP tends to provide additional routes for liquidity to circulate more efficiently. By contrast, 


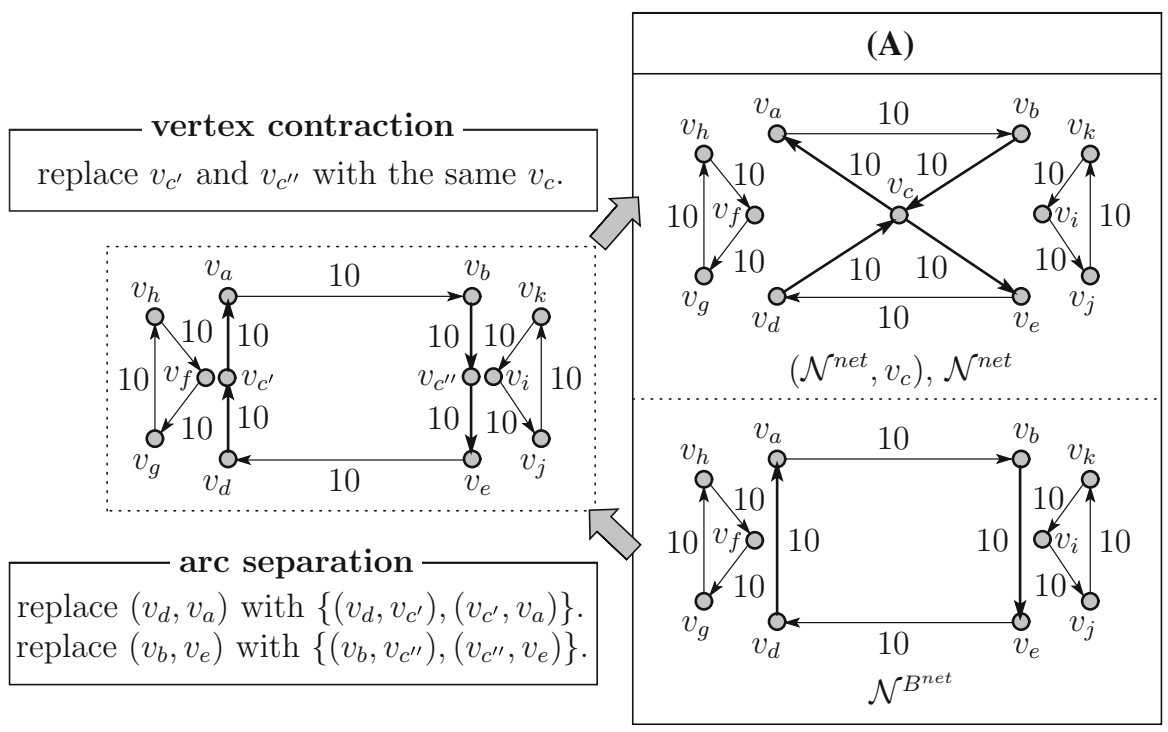

Fig. 22 Illustration of the central routing effect (1)

in the bad environment, additional CCP serves as an additional stop for liquidity, which always increases liquidity needs.

We illustrate the intuition for the proof using Figs. 22 and 23. Given financial system $\left(\mathcal{N}^{n e t}, v_{c}\right)$, which is shown in the upper part of the box in each figure, the same $N^{n e t}$ shows the net-out CCP network. In each of the two figures, bilateral network $\mathcal{N}^{B_{n e t}}$ is shown in the lower part in the box. In the proof, we define a procedure to derive $\mathcal{N}^{n e t}$ from arbitrary $\mathcal{N}^{B_{n e t}}$. The procedure consists of two operations on the relevant network. As illustrated in the figures, the operations are referred to as arc separation and vertex contraction, for which the relevant concrete operations are described in each figure, and the definitions are formally stated in the appendix. In the relevant procedure, the arc separation serves to add additional vertices, while the vertex contraction reduces the added vertices by merging them into one vertex.

As formally shown in the appendix, in the bad environment, the arc separation always increases the total liquidity needs in proportion to the number of added vertices. Although the proceeding vertex contraction serves to reduce the total liquidity needs by reducing the number of vertices, the effect by the preceding arc separation is always larger. In the good environment, the arc separation has no effect on the total liquidity needs. The vertex contraction always serve to weakly decrease the total liquidity needs, by enlarging the possible route for each liquidity to circulate. Observe that in Fig. 22, the vertex contraction fails to enlarge the relevant route, while in Fig. 23, the vertex contraction successfully enlarges the route by connecting the two cycles.

In order to observe the quantitative aspect of the central routing effect, we define a class of basic net-out financial systems, which includes financial systems shown in the upper row in Fig. 24. We let a triangle refer to a cycle that is expressed with three different vertices $\left\{v_{a}, v_{b}, v_{c}\right\}$ and three $\operatorname{arcs}\left\{\left(v_{a}, v_{b}\right),\left(v_{b}, v_{c}\right),\left(v_{c}, v_{a}\right)\right\}$. 


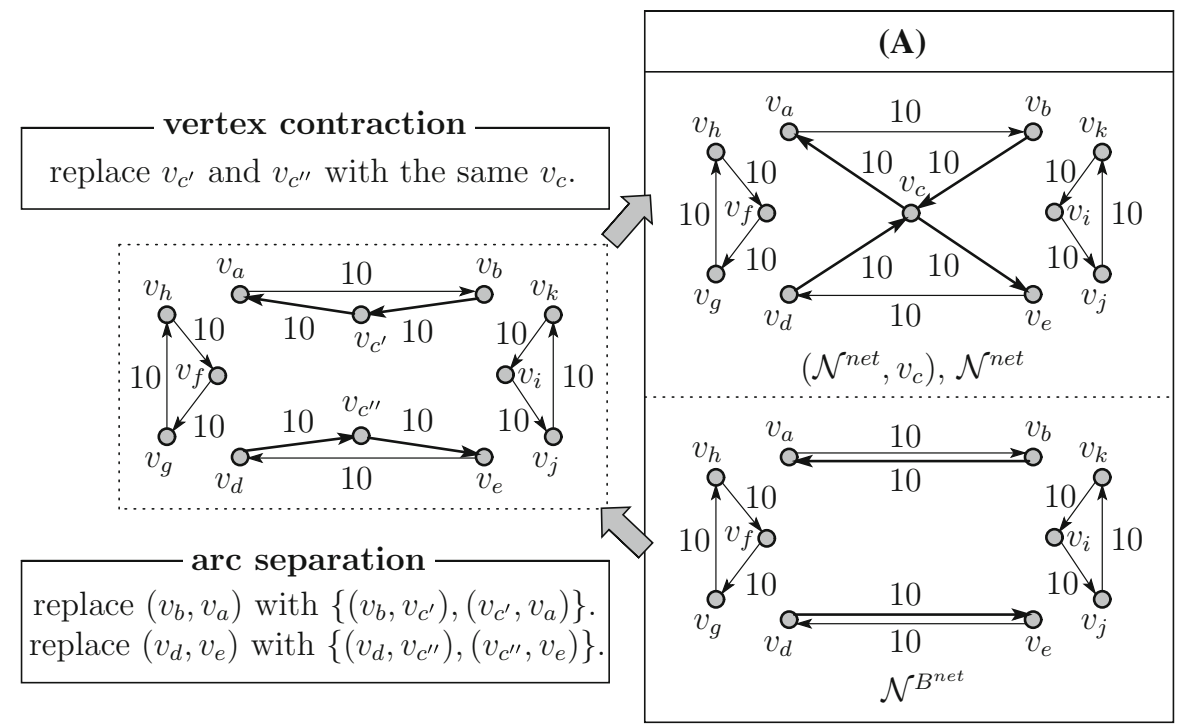

Fig. 23 Illustration of the central routing effect (2)

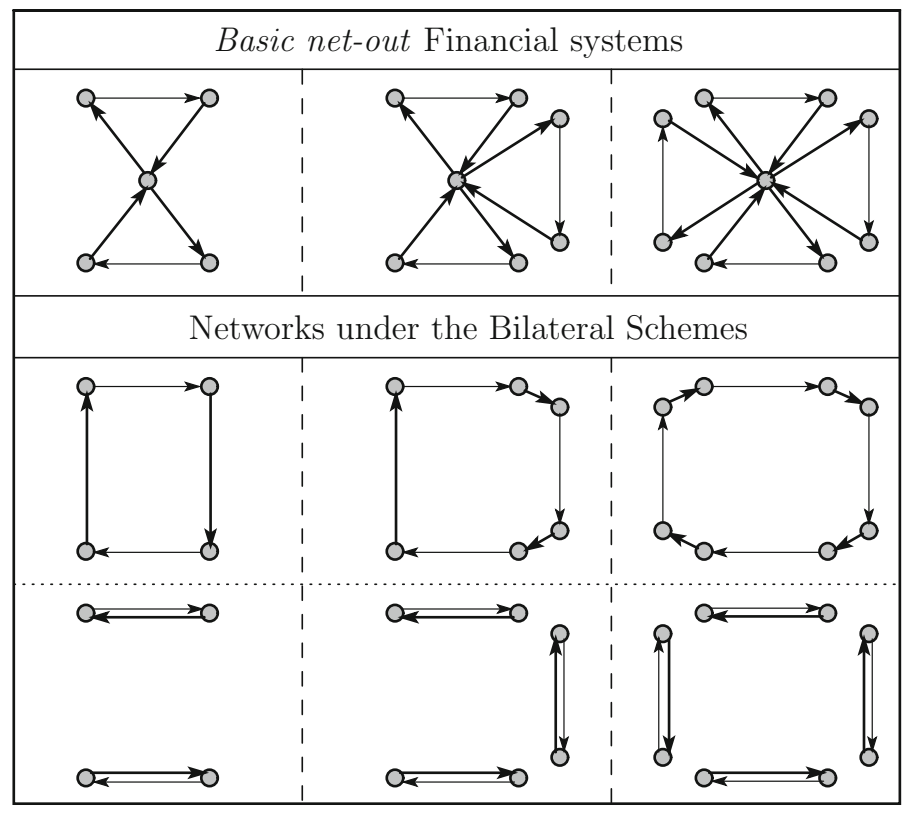

Fig. 24 Examples of basic net-out financial systems. Weights on the arcs are not shown. The upper row shows three basic net-out financial systems in which the center vertex is the hypothetical entity for each. In the middle and lower rows for each column, we show the relevant bilateral networks 
Definition 1 Basic net-out financial system

A financial system $\left(\mathcal{N}, v^{*}\right)$ is a basic net-out financial system, when

(i) it consists of triangles, and

(ii) $v^{*}$ is included in every triangle, and every triangle is connected with each other only with vertex $v^{*}$.

Theorem 3 The central routing effect: Basic net-out financial system

Given basic net-out financial system $\left(\mathcal{N}^{n e t}, v^{*}\right)$ with $J \geq 2$ triangles, let $f^{m}$ denote the unit price of the target security. For arbitrary bilateral network $\mathcal{N}^{B_{\text {net }}}$ for $\left(\mathcal{N}^{\text {net }}, v^{*}\right)$, we obtain

(i) $-J f^{m} \leq x^{\max }\left(\mathcal{N}^{B_{n e t}}\right)-x^{\max }\left(\mathcal{N}^{\text {net }}\right) \leq-f^{m}$.

(ii) $0 \leq x^{\min }\left(\mathcal{N}^{B_{n e t}}\right)-x^{\min }\left(\mathcal{N}^{n e t}\right) \leq(J-1) f^{m}$.

\section{Proof See Appendix 7.4.}

Figure 24 easily confirms each of the lower bound and upper bound of the abovementioned results. Observe that each network shown in the middle row in the figure consists of one cycle, while each network in the bottom row consists of $J$ number of cycles with $J$ as the number of the relevant triangles. In the bad environment, the smallest negative effect of the central routing effect is attained by networks in the middle row, while the largest negative effect is attained by those in the bottom row. This is because for each cycle, exactly one vertex is exempt from inputting additional liquidity in the bad environment, and thus, a larger number of cycles means that more vertices are exempt, given a fixed number of vertices in total. In the good environment, the middle row shows no effect of the central routing effect, while the bottom row shows the largest positive effect. It is easy to observe that a larger number of cycles means less efficient circulation of liquidity, since at least one vertex in each cycle needs to input liquidity.

Focusing on the bad environment, part (i) of Theorem 3 quantifies the range of the negative effect of the central routing effect for the relevant class. In Sect. 5.4, we argue about how much netting efficiency is required in order to cancel out the negative effect.

\subsection{Central netting effect}

The central netting effect is also clarified through the operations of arc separation and vertex contraction. We first illustrate this point using Fig. 25. The upper part of the box shown in the figure is the same as that in Fig. 20, which illustrates the (P1) procedure. Figure 25 illustrates that the (P1) procedure is replicated with the operations of the reverse of arc separation and the reverse of vertex contraction. From the opposite view, suppose that the (P1) procedure is derived $\mathcal{N}^{B_{n e t}}$ from $\mathcal{N}^{B}$, as shown in Fig. 20. Then, Fig. 25 illustrates that we conversely derive $\mathcal{N}^{B}$ from $\mathcal{N}^{B_{\text {net }}}$ by applying vertex contraction and arc separation in this sequence. The effect of the combination of arc separation and vertex contraction is already examined in Sect. 5.2.

One difference from the central routing effect is that another operation is relevant for the case of the central netting effect, which is illustrated in Fig. 26. The figure 


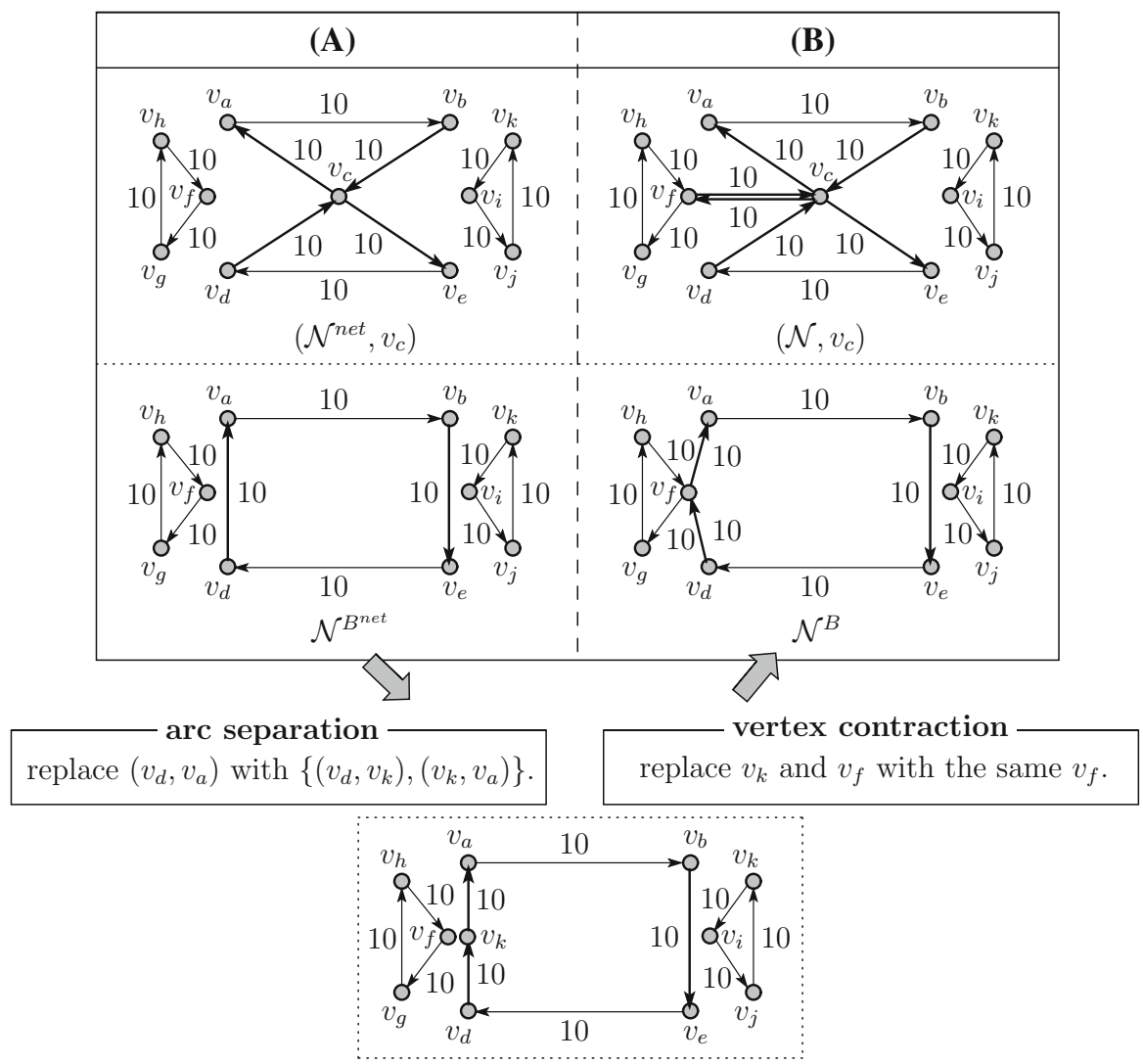

Fig. 25 Illustration of the central netting effect (1)

shows two financial systems $(A)$ and $(C)$ and each relevant bilateral network. When we examine the effect in the direction from $\mathcal{N}^{B_{n e t}}$ to $\mathcal{N}^{B}$, the operation is referred to as cycle addition, for which the concrete operation is described in the figure and the definition is formally stated in the appendix.

The central netting effect is not essentially as simple as the central routing effect is, since cycle addition is also relevant. Still, the next theorem shows that the central netting effect is rather simply stated in the bad environment.

Theorem 4 Central netting effect in the bad environment

The central netting effect is always strictly positive in the bad environment.

Formally, given financial system $\left(\mathcal{N}, v^{*}\right)$, for any bilateral network $\mathcal{N}^{B}$, by taking corresponding bilateral network $\mathcal{N}^{B_{n} \text { et }}$ through the $(P 1)$ procedure, we obtain

$$
x^{\max }\left(\mathcal{N}^{B}\right)-x^{\max }\left(\mathcal{N}^{B_{n e t}}\right)>0 .
$$

Proof See Appendix 7.5. 


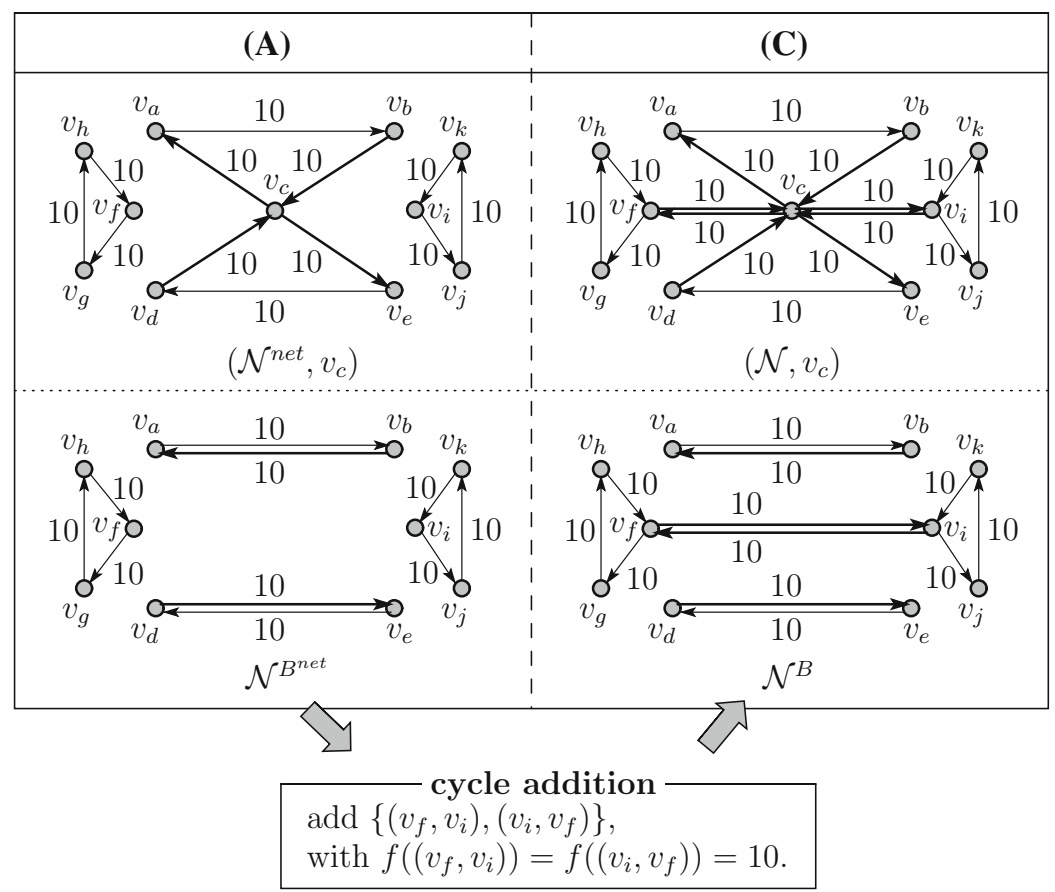

Fig. 26 Illustration of the central netting effect (2)

The intuition of the proof is simple. Regarding the combination of the operations of arc separation and vertex contraction, we have already confirmed that it strictly increases the total liquidity needs in the bad environment. In addition, cycle addition serves to generate additional liquidity needs, and thus, the total liquidity needs also strictly increase in the bad environment. Since the central netting effect is examined in the opposite direction, the central netting effect serves to strictly decrease the total liquidity needs in the bad environment.

So far, we have confirmed that, in the bad environment, the central routing effect is negative (part (i) of Theorem 2 in Sect. 5.2), while the central netting effect is positive (Theorem 4). According to this, larger netting efficiency tends to improve the total effect, but insufficient netting efficiency leads to a negative effect in total. In the next subsection, we argue the quantitative aspect with respect to the extent of netting efficiency required to cancel out the negative effect caused by the central routing effect.

In the good environment, it is possible for the central netting effect to be positive or negative, as illustrated in the previous section. Remember that the combination of the operations of arc separation and vertex contraction weakly decreases the total liquidity needs in the good environment, as mentioned in the central routing effect. However, both directions of the effect of cycle addition are possible. For the specific example shown in Fig. 26, cycle addition decreases the total liquidity needs in the good environment. The intuition is that the added cycle serves to connect the separated cycles, and thus, serves to enlarge the route for liquidity to circulate. Specifically for 


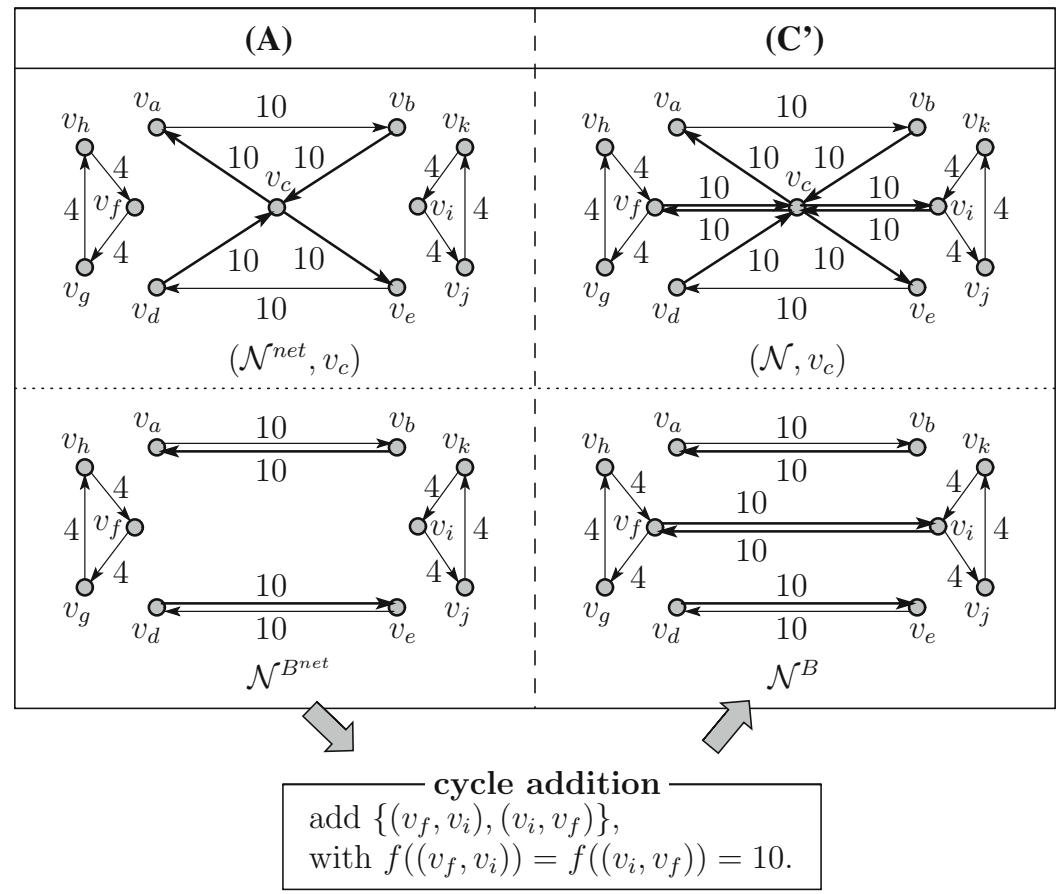

Fig. 27 Illustration of the central netting effect (3)

the example, we obtain $x^{\min }\left(\mathcal{N}^{B}\right)-x^{\min }\left(\mathcal{N}^{B_{n e t}}\right)=10 * 3-10 * 4=-10<0$. An opposite effect of cycle addition is illustrated in Fig. 27, in which financial system (C') is different from (C) in that the weights of the arcs for the two cycles placed on the left and right are 4 instead of 10 . The corresponding bilateral network shown on the lower-right is derived from the bilateral network shown on the lower-left by adding a cycle with the weight 10 . Although adding the cycle still serves to enlarge the route for liquidity to circulate, additional liquidity is now required for the added cycle. Thus, cycle addition in this example increases the total liquidity needs in the good environment, which means that the central netting effect is positive. Specifically for this example, we obtain $x^{\min }\left(\mathcal{N}^{B}\right)-x^{\min }\left(\mathcal{N}^{B_{n e t}}\right)=10 * 3-(10 * 2+4 * 2)=2>0$. This indicates that the central netting effect tends to be negative when each obligation settled by the CCP is relatively small compared to each obligation settled without the CCP.

For our formal statements of the relevant results, we prepare the terminology of isolated cycles. Given financial system $\left(\mathcal{N}, v^{*}\right)$ with network $\mathcal{N}=\langle V, A, f\rangle$, an isolated cycle is a cycle with a set of vertices $V^{\prime} \subset V \backslash v^{*}$ such that $V^{\prime}$ constitute a cycle in which each vertex $v \in V^{\prime}$ is not included in more than two $\operatorname{arcs}$ within $A$, thereby an isolated cycle consists of no more than one cycle, there is no more than one arc for any pair of vertices within $V^{\prime}$, and there is no other vertex $v^{\prime} \in V \backslash V^{\prime}$ that constitutes an arc with a vertex $v \in V^{\prime}$. For a given balanced network, we refer to the weight of each isolated cycle as the weight of an arbitrary arc in each cycle, since any 
arc in the same isolated cycle has the same weight. Specifically, for financial system $\left(\mathcal{N}^{n e t}, v_{c}\right)$ shown on the upper-left in Fig. 26, there are two isolated cycles, one with vertices $\left\{v_{f}, v_{g}, v_{h}\right\}$, and the other with $\left\{v_{i}, v_{j}, v_{k}\right\}$, in which the weight of each cycle is 10 .

We compare a financial system that includes isolated cycles, with its corresponding financial system in which the isolated cycles are connected to the hypothetical entity. An example is shown in Fig. 26, in which each of the two isolated cycles in $\left(\mathcal{N}^{n e t}, v_{c}\right)$ is connected to the hypothetical entity $v_{c}$ in $\left(\mathcal{N}, v_{c}\right)$. Specifically, let $v^{*}$ denote the hypothetical entity, and take arbitrary vertex $v$ for an isolated cycle. Then, add a pair of $\operatorname{arcs}\left\{\left(v, v^{*}\right),\left(v^{*}, v\right)\right\}$. Add a pair of arcs for every isolated cycle in this manner. The weight of each added arc is endowed by the given unit price of the target-security.

The next theorem shows the central netting effect for a class of financial systems that include connected isolated cycles. The theorem reveals the quantitative aspect of the central netting effect both in the good and bad environment, which is further examined in combination with the central routing effect in the next subsection.

Theorem 5 Central netting effect: quantitative aspect

Given net-out financial system $\left(\mathcal{N}^{n e t}, v^{*}\right)$ in which there exist $2 K$ number of isolated cycles $\left\{V_{1}, V_{2}, . ., V_{2 K}\right\}$ with integer $K \geq 1$, and there is at least a pair of arcs $\left\{\left(v, v^{*}\right),\left(v^{*}, v^{\prime}\right)\right\}$ such that $v \neq v^{\prime}$ and $v, v^{\prime} \notin\left\{V_{k}\right\}_{k=1,2, \ldots, 2 K}$, take another financial system $\left(\mathcal{N}, v^{*}\right)$ by connecting every isolated cycle to the hypothetical entity $v^{*}$. Denote the unit price of the target security as $f^{m}$, and denote the weight of each cycle indexed with $k=1,2, . ., 2 K$ as $f^{k}$.

Then, for arbitrary bilateral network $\mathcal{N}^{B}$ of the financial system $\left(\mathcal{N}, v^{*}\right)$, by taking corresponding net-out bilateral network $\mathcal{N}^{B_{\text {net }}}$ through the $(P 1)$ procedure, we obtain

(i) $K f^{m} \leq x^{\max }\left(\mathcal{N}^{B}\right)-x^{\max }\left(\mathcal{N}^{B_{n e t}}\right) \leq 2 K f^{m}$, and

(ii) $-\Sigma_{1}^{2 K} \min \left(f^{k}, f^{m}\right) \leq x^{\min }\left(\mathcal{N}^{B}\right)-x^{\min }\left(\mathcal{N}^{B_{n e t}}\right)$.

Furthermore, when $f^{m}$ is sufficiently small such that $f^{m} \leq f^{k}$ for every $k=$ $1,2, \ldots, 2 K$, we obtain

(ii') $-2 K f^{m} \leq x^{\min }\left(\mathcal{N}^{B}\right)-x^{\min }\left(\mathcal{N}^{B_{n e t}}\right) \leq-K f^{m}$.

Proof See Appendix 7.6.

The top row in Fig. 28 shows a financial system with four isolated cycles and a corresponding financial system with connected isolated cycles. The middle and bottom rows show relevant bilateral networks such that each network on the left is derived by the $(\mathrm{P} 1)$ procedure for the network on the right in the same row.

Observe that for the bilateral networks in the middle row, cycle addition is not relevant for the relevant (P1) procedure, while only cycle addition is relevant for those in the bottom row. For result (i) of Theorem 5, we observe that the largest positive effect in the bad environment is attained for the bilateral networks shown in the middle row, while the smallest positive effect is attained for the bilateral networks in the bottom row. The intuition is that in the relevant (P1) procedure, liquidity needs reduced by the reverse of cycle addition is smaller than those reduced by corresponding combination of reverse vertex contraction and reverse arc separation. 


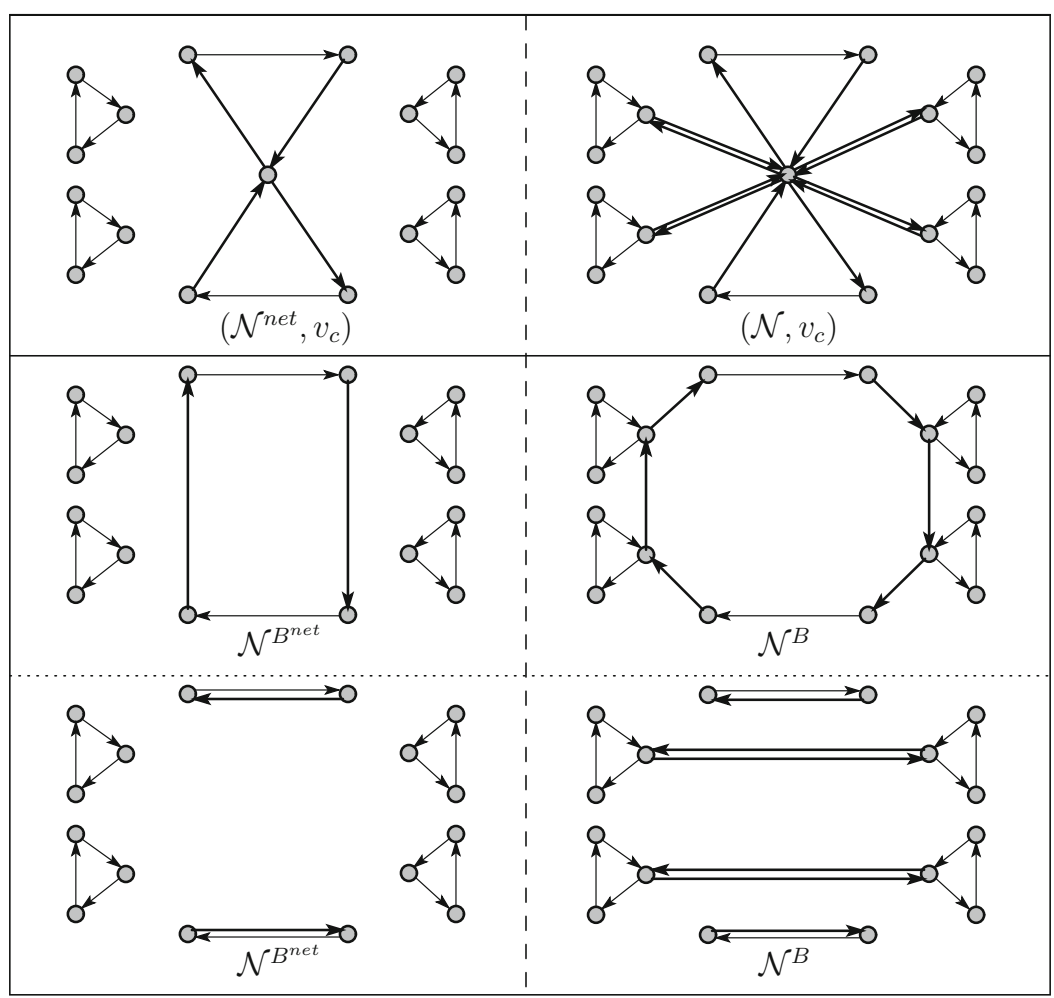

Fig. 28 Illustration of the central netting effect (4). We do not show relevant weights for the arcs. Each weight can be supposed as 10 , or other values that satisfy our assumptions. For financial system $\left(\mathcal{N}, v_{c}\right)$ shown on the upper right, the corresponding financial system $\left(\mathcal{N}^{n e t}, v_{c}\right)$ is shown on the upper left. For each financial system, the lower part of the figure shows two bilateral networks. For each network denoted as $\mathcal{N}^{B}$, the left network denoted as $\mathcal{N}^{B_{n e t}}$ on the same height is derived by the (P1) procedure

For result (ii), the largest negative effect in the good environment is attained for the bilateral networks shown in the middle row. For the intuition, in the good environment, the total liquidity needs become larger as the number of cycles increases. Actually, for networks shown in the middle row, the number of cycles increases by 4 (from 1 to 5), while for networks in the bottom row, the number increases by 2 (from 4 to 6).

For result (ii'), the smallest possible negative effect is attained for networks shown in the bottom of the figure. There, effectively two cycles are eliminated, and this has a negative effect when the weights of the cycles are sufficiently small, which is ensured by the added condition for the result.

\subsection{Netting efficiency of a CCP and total effect}

To examine the total effect of a CCP, we examine a specific class of financial systems, in which a basic net-out financial system (defined in Sect. 5.2) is combined with isolated cycles connected to the hypothetical entity. For example, financial system (C) 
in Fig. 26, (C') in Fig. 27, and a financial system shown on the upper right in Fig. 28 are within the class. We refer to a financial system within the class as a $(J, 2 K)$ financial system with integers $J \geq 2$ and $K \geq 1$, in which $J$ triangles constitute a basic net-out financial system, and $2 K$ isolated cycles are connected to the hypothetical entity. Both financial systems $(C)$ and $\left(C^{\prime}\right)$ are $(2,2)$ financial systems, while the financial system shown on the upper right in Fig. 28 is a $(2,4)$ financial system.

We refer to netting efficiency for a given financial system, as the ratio of the amount of obligations that are eventually offset to the total amount of obligations with respect to the hypothetical entity. Netting efficiency for a $(J, 2 K)$ financial system is derived as $\frac{2 K}{J+2 K}$. Thus, note that netting efficiency for the financial system shown on the upper right in Fig. 28 is two-thirds, which is shown as the threshold value for the direction of the total effect of adding a CCP in the bad environment.

According to part (i) of the following Theorem 6, for the specified class, the netting efficiency of a CCP must be larger than two-thirds in order for the total effect of a $\mathrm{CCP}$ to be always positive in the bad environment.

According to part (ii), the worst of the total effect of adding a CCP in the good environment is always negative, while it is possible for the best of the total effect to be positive. Furthermore, according to part (ii'), when netting efficiency is larger than two-thirds and each obligation settled by the added CCP is relatively small, then the total effect of adding the CCP in the good environment is always negative.

Theorem 6 For given $(J, 2 K)$ financial system $\left(\mathcal{N}, v^{*}\right)$ with isolated cycles $\left\{V_{1}, V_{2}\right.$, $\left.\ldots, V_{2 K}\right\}$, denote the unit price of the target security as $f^{m}$, and denote the weight of cycle $V_{k}$ as $f^{k}$ for $k=1,2, \ldots, 2 K$. Then, for arbitrary bilateral network $\mathcal{N}^{B}$ of financial system $\left(\mathcal{N}, v^{*}\right)$, we obtain

(i) $x^{\max }\left(\mathcal{N}^{B}\right)-x^{\max }\left(\mathcal{N}^{\text {net }}\right) \geq 0$ if and only if $J \leq K$, and

(ii) $-\Sigma_{1}^{2 K} \min \left(f^{k}, f^{m}\right) \leq x^{\min }\left(\mathcal{N}^{B}\right)-x^{\min }\left(\mathcal{N}^{n e \bar{t}}\right)$.

Furthermore, suppose $J \leq K$. When $f^{m}$ is sufficiently small such that $f^{m} \leq f^{k}$ for every $k=1,2, \ldots, 2 K$, then, we obtain

(ii') $x^{\min }\left(\mathcal{N}^{B}\right)-x^{\min }\left(\mathcal{N}^{n e t}\right) \leq-f^{m}$.

\section{Proof See Appendix 7.7.}

The results are rather simply understood in light of our decomposition. We illustrate the intuition using Fig. 28. For financial system $\left(\mathcal{N}, v_{c}\right)$ shown on the top-right of the figure, observe that in the bad environment, the smallest positive central netting effect is attained for bilateral networks shown in the bottom row. Then, the largest negative central routing effect in the bad environment is attained for the bilateral networks between the bottom-left network and the net-out CCP network shown on the top-left. Thus, for result (i), the largest negative total effect in the bad environment is derived by adding the relevant effects, in which the relevant values are explicitly shown in part (i) of Theorem 3 and part (i) of Theorem 5.

For result (ii) regarding the lower bound of the total effect (largest possible negative effect), in the good environment, the largest negative central netting effect is attained for the bilateral networks shown in the middle row, and the smallest positive central 
routing effect, which is zero, is again attained for the bilateral networks between the bottom-left network and the net-out CCP network shown on the top-left. The relevant values are derived by combining part (ii) of Theorem 3 and part (ii) of Theorem 5 .

For result (ii') regarding the upper bound of the total effect, under the added conditions, the smallest negative central netting effect is attained for the bilateral networks shown in the bottom row, and the largest positive central routing effect is attained between the bottom-left network and the net-out CCP network. The relevant values are derived by combining part (ii) of Theorem 3 and part (ii') of Theorem 5 .

\subsection{Policy implications}

Our analysis shows that even during times of financial distress, utilization of a CCP is not unconditionally successful in reducing overall liquidity needs. This is because the added CCP itself tends to demand liquidity, typically in the form of margin for derivative trades. Still, multilateral netting provided by the CCP tends to decrease the overall liquidity needs. Thus, adding a CCP reduces the liquidity needs in total as long as the netting efficiency is sufficiently large. Conversely, this indicates that a CCP should not be used if netting efficiency is expected to be sufficiently small. Although the threshold netting efficiency depends on each network topology, our analysis indicates that the threshold is not trivially low, suggesting two-thirds as one benchmark value for a relevant policy.

Furthermore, our analysis indicates that operating a CCP after an economy is no longer in financial distress could be costly from the perspective of overall liquidity needs. Especially when an economy is far from financial distress, and liquidity could be circulated in a highly efficient manner, multilateral netting by the CCP could hinder the efficient circulation of liquidity. A negative effect of a CCP is more likely when each contracted amount for trades settled by the CCP is relatively small. Thus, our analysis indicates that operating a CCP that settles trades in relatively small amounts is rather costly even when the expected netting efficiency is sufficiently high.

In total, the analysis indicates the possible merit in the flexible utilization of a CCP only during times of financial distress. For trades that are settled by a CCP with high netting efficiency but each trade amount is relatively small, flexible utilization has an advantage over inflexible utilization of a CCP, in that the CCP operates regardless of the state of the economy. Nevertheless, this needs to be examined in combination with other relevant costs, which are not considered in this study.

\subsection{Remark: specification of a CCP}

In our analysis, we assume there is no difference between a CCP and other financial institutions regarding the manner in which liquidity is demanded. However, this is not necessarily the case in reality. Here, we take up two types of CCP specifications and argue about the implications for our results. Each specification can be expressed by adding constraints to our liquidity problems.

The first specification requires that the CCP never inputs its own liquidity to settle its obligations. This is a probable setting to reduce the liquidity cost born by the CCP. 
We call such a CCP "passive." For a given financial system, for the CCP to be "passive" in the relevant settlements, the liquidity problems introduced in Sect. 2.4 are altered as follows.

\section{(Liquidity problems with passive CCP)}

Given network $\langle V, A, f\rangle$ with CCP $v^{*} \in V$, take one-to-one mapping $s: A \rightarrow$ $\{1,2, \ldots,|A|\}$ and associated $\left\{p_{v}(s)\right\}_{v \in V \backslash v^{*}}$ such that,

$$
\begin{aligned}
& \min (\max )_{s} \sum_{v \in V} p_{v}(s) \\
& \text { s.t., } p_{v^{*}}(s)=0 \quad \text { for any } s: A \rightarrow\{1,2, \ldots,|A|\}
\end{aligned}
$$

The last line is the added constraint. It is clear that the altered minimization problem yields the same value for the original minimization problem. To observe this, note that there are at least two vertices for each cycle, and only one vertex is sufficient to input liquidity for each cycle. Although it is more delicate, the altered maximization problem also yields the same value as the original maximization problem. Observe that, for the case of maximization, at least one vertex is exempt from inputting liquidity for each cycle. Since only one CCP is assumed for the relevant network, it is always possible to allow the CCP be such a vertex. ${ }^{13}$

Next, we further assume that CCPs must receive all the relevant payments before making any payments. This would be a reasonable specification to guarantee that CCPs are passive. We say that such a CCP is both passive and synchronous. For the CCP to be passive and synchronous in the relevant settlements, our liquidity problems are now altered as follows.

\section{(Liquidity problems with passive and synchronous CCP)}

Given network $\langle V, A, f\rangle$ with CCP $v^{*} \in V$, take one-to-one mapping $s: A \rightarrow$ $\{1,2, \ldots,|A|\}$ and associated $\left\{p_{v}(s)\right\}_{v \in V}$ such that,

$$
\begin{aligned}
& \min (\max )_{s} \sum_{v \in V} p_{v}(s), \\
& \text { s.t., } \max _{\left(v^{\prime}, v^{*}\right) \in A} s\left(\left(v^{\prime}, v^{*}\right)\right)<\min _{\left(v^{*}, v^{\prime \prime}\right) \in A} s\left(\left(v^{*}, v^{\prime \prime}\right)\right) .
\end{aligned}
$$

The added condition is restrictive in which the CCP makes any of its payments after it has received all the payments. It is clear that the altered minimization problem tends to yield larger value compared to the value for the original minimization problem. This is because the synchronous aspect of the CCP tends to hinder efficient possible liquidity circulation. By contrast, the altered maximization problem yields the same value for the original maximization problem. This is because the maximum value for

\footnotetext{
13 Note that when there is more than one "passive" CCP, the overall required liquidity in the bad environment tends to be smaller. This means that adding more CCPs tends to have a smaller negative effect per CCP. Still, the smaller effect is not probable when the added CCP is not sufficiently close to the existent CCPs in the relevant network.
} 
the original problem is attained in such order as specified by synchronous supposition, and thus, the condition does not effectively serve as any additional constraint.

We confirm that passive and synchronous CCP ends up exacerbating the negative effect in the good environment. This merely strengthens our relevant results regarding the disadvantage of adding a CCP, and accordingly suggests even larger merit of flexible utilization of a CCP.

Note that it is possible for the specifications of the CCP to affect the central routing effect but they never affect the central netting effect. This is generally true for arbitrary specification of the CCP, since the CCP is not explicitly relevant for the central netting effect. This observation shows another aspect of the analytical usefulness of our decomposition of the effect.

\section{Concluding remarks}

Utilization of CCPs for derivative trades has become a trend after the recent financial crisis. The possible disadvantages of CCPs need to be examined carefully in combination with their advantages. The existent literature has well clarified how CCPs possibly increase counterparty risk, by affecting relevant exposure. However, there has been little investigation on how CCPs possibly affect the overall liquidity needs. Such investigation is crucial, since significant liquidity needs could lead to liquidity shortage in times of financial distress, which could cause firesales and even "dominos" of bankruptcy. The difficulty of such investigation has its root in the dynamic nature of liquidity needs, which is essentially different from the static nature of corresponding exposure or obligations.

The presented model captures the static nature of the formed obligations through the concept of network. Then, the model captures the dynamic nature of liquidity needs through the relative order of payments regarding each network. This approach enables us to differentiate economic states regarding how much liquidity tends to be needed. We especially argue about liquidity needs during times of financial distress and when an economy is far from such distress. The effect of adding a CCP to the overall liquidity needs is shown to be decomposed into two effects: the central routing effect and the central netting effect. The decomposition is captured with respect to network topology, specifically through several operations in each network.

Our analysis primarily reveals the qualitative nature of each decomposed effect. Observing that the quantitative nature of the effect essentially depends on each network topology, the analysis also provides quantitative results for a specific class of networks. Further investigation into the quantitative aspects remains for future research.

Beyond the specific topic of CCPs, our analytical approach sheds new light on relevant liquidity issues. For example, liquidity is crucial in the debate about the propagation of loss of a financial institution through an interconnected network of contracts. However, most existent literature focuses on the effects on balance sheets of the relevant institutions, ignoring how much liquidity is available at each moment. This necessarily ignores bankruptcy arising from the liquidity problem, which is important in reality. Our model and analysis sets a foundation for examining dynamics of liquidity transfers, and have wide potential in the relevant applications. 
Acknowledgements I would like to thank the editor and the anonymous referees for their careful reviews on an earlier version of this paper. This work was partly supported by JSPS KAKENHI Grant Number 16H02009, and by the European Union Seventh Framework Programme (FP7/2007-2013) under grant agreement $n^{\circ}$ PIRSES-GA-2012-317767.

Open Access This article is distributed under the terms of the Creative Commons Attribution 4.0 International License (http://creativecommons.org/licenses/by/4.0/), which permits unrestricted use, distribution, and reproduction in any medium, provided you give appropriate credit to the original author(s) and the source, provide a link to the Creative Commons license, and indicate if changes were made.

\section{Appendix}

\subsection{Relevant definitions and preliminary results}

The following definitions and results are from Hayakawa (2014).

For network $\langle V, A, f\rangle$, we define arc separation on $\left(v, v^{\prime}\right) \in A$ as the following operations to yield another network $\left\langle V^{\prime}, A^{\prime}, f^{\prime}\left\langle\right.\right.$ : (i) remove the arc $\left(v, v^{\prime}\right)$, then, (ii) add new vertex $v^{\prime \prime}$ and two $\operatorname{arcs}\left(v, v^{\prime \prime}\right),\left(v^{\prime \prime}, v^{\prime}\right)$ such that $f^{\prime}\left(\left(v, v^{\prime \prime}\right)\right)=f^{\prime}\left(\left(v^{\prime \prime}, v^{\prime}\right)\right)=$ $f\left(\left(v, v^{\prime}\right)\right)$.

In addition, we define vertex contraction on $v, v^{\prime} \in V$ as the operation to merge $v$ and $v^{\prime}$ into $v$, or in other words, to replace $v^{\prime}$ with $v$ for the relevant arcs. Furthermore, we define cycle addition as the operation to add new arcs $A^{\prime}$ either with or without new vertices, so that $A^{\prime}$ constitutes a cycle.

\section{Lemma 1 Arc separation}

Given balanced network $\mathcal{N}=\langle V, A, f\rangle$, let $\mathcal{N}^{\prime}$ denote a network derived by arc separation on arbitrary $a \in A$. Then, we obtain

$$
\begin{aligned}
x^{\min }\left(\mathcal{N}^{\prime}\right) & =x^{\min }(\mathcal{N}) \text { and } \\
x^{\max }\left(\mathcal{N}^{\prime}\right) & =x^{\max }(\mathcal{N})+f(a) .
\end{aligned}
$$

\section{Lemma 2 Vertex contraction}

(i) Given balanced network $\mathcal{N}=\langle V, A, f\rangle$, let $\mathcal{N}^{\prime}$ denote a network derived by vertex contraction on two arbitrary vertices $v, v^{\prime} \in V$. Then, we obtain

$$
\begin{aligned}
& x^{\min }\left(\mathcal{N}^{\prime}\right) \leq x^{\min }(\mathcal{N}) \text { and } \\
& x^{\max }\left(\mathcal{N}^{\prime}\right) \leq x^{\max }(\mathcal{N}) .
\end{aligned}
$$

(ii) Furthermore, take $K$ isolated cycles, and call each cycle $k=1,2, \ldots, K$. Let $f^{k}$ denote the weight of cycle $k$, for $k=1,2, \ldots, K$. Let cycle 1 include $L>K$ number of vertices, and arbitrarily denote the vertices as $v_{1}, v_{2}, . ., v_{L}$. For $k=2,3, \ldots, K$, adopt vertex contraction between one vertex of cycle $k$ and vertex $v_{k}$ of cycle 1 . We derive a network such that cycle 1 is now connected to every other cycle $k>1$ with each different vertex $v_{k}$. Denote the derived network 
as $\mathcal{N}^{\prime}$. Then, we obtain

$$
\begin{aligned}
& x^{\min }\left(\mathcal{N}^{\prime}\right)=x^{\min }(\mathcal{N})-\sum_{k=2}^{K} \min \left(f^{1}, f^{k}\right) \text { and } \\
& x^{\max }\left(\mathcal{N}^{\prime}\right)=x^{\max }(\mathcal{N})
\end{aligned}
$$

(iii) Alternatively, take one isolated cycle with weight $f^{m}$, in which there are more than four vertices included. Take two vertices $v, v^{\prime}$ included in the cycle such that there is no arc between them. Let $\mathcal{N}^{\prime}$ denote a network derived by vertex contraction on $v, v^{\prime}$. Then, we obtain

$$
\begin{aligned}
& x^{\min }\left(\mathcal{N}^{\prime}\right)=x^{\min }(\mathcal{N}) \quad \text { and } \\
& x^{\max }\left(\mathcal{N}^{\prime}\right)=x^{\max }(\mathcal{N})-f^{m} .
\end{aligned}
$$

For part (ii) in Lemma 2, an example network is shown on the middle-right in Fig. 28, where the center cycle with 8 vertices is interpreted as cycle 1, which is connected to each of the other 4 cycles.

\section{Lemma 3 Cycle addition}

(i) Given balanced network $\mathcal{N}=\langle V, A, f\rangle$, let $\mathcal{N}^{\prime}$ denote a network derived by cycle addition with arbitrary cycle $A^{\prime}$. Then, we obtain

$$
x^{\max }\left(\mathcal{N}^{\prime}\right)>x^{\max }(\mathcal{N}) .
$$

(ii) Furthermore, take $K$ isolated cycles, each with weight $f^{1} \leq f^{2} \leq, \cdots, \leq$ $f^{K}$. Arbitrarily take one vertex $v_{k}$ for each cycle $k=1, \overline{2}, \ldots, \bar{K}$. Add $\bar{a}$ cycle $\left\{\left(v_{1}, v_{2}\right),\left(v_{2}, v_{3}\right), \ldots,\left(v_{K}, v_{1}\right)\right\}$ with the same weight $f\left(\left(v_{1}, v_{2}\right)\right)=$ $f\left(\left(v_{2}, v_{3}\right)\right)=\cdots=f\left(\left(v_{K}, v_{1}\right)\right)=f^{m}$. We derive a network such that the added cycle is connected to every existent cycles with each different vertex. Denote the derived network as $\mathcal{N}^{\prime}$. This yields

$$
\begin{aligned}
x^{\min }\left(\mathcal{N}^{\prime}\right)= & x^{\min }(\mathcal{N})+f^{m}-\left(\sum_{k=1}^{K} \min \left(f^{k}, f^{m}\right)\right) \\
& \text { and thus, } x^{\min }\left(\mathcal{N}^{\prime}\right)>x^{\min }(\mathcal{N}) \text { iff } f^{m}>\sum_{k=1}^{K} f^{k} . \\
x^{\max }\left(\mathcal{N}^{\prime}\right)= & x^{\max }(\mathcal{N})+(K-1) f^{m} .
\end{aligned}
$$

\subsection{Proof of Theorem 1}

\section{Proof of (i)}

The (P1) procedure yields a unique network, since the order of taking offsettable pairs does not change the derived network. It is evident that each derived network is a bilateral network of $\left(\mathcal{N}^{n e t}, v^{*}\right)$. 


\section{Proof of (ii)}

For financial system $\left(\mathcal{N}=\langle V, A, f\rangle, v^{*}\right)$, take arcs owed to $v^{*}$ and owed by $v^{*}$, which are each denoted as $A_{t o}, A_{b y}$. We derive $A_{t o}^{\prime}, A_{b y}^{\prime}$ by removing arcs that constitute any offsettable pair from each of $A_{t o}, A_{b y}$. Observe that arbitrary one-to-one matching between $A_{t o}$ and $A_{b y}$ yields a set of paths from $A_{t o}^{\prime}$ to $A_{b y}^{\prime}$, and cycles formed by offsettable pairs. Remove all those cycles, and then, for each of the remained path, let the starting arc be matched with the ending arc. This yields a one-to-one matching between $A_{t o}^{\prime}, A_{b y}^{\prime}$, and this is exactly what the (P1) procedure does. Thus, it is evident that every possible one-to-one matching between $A_{t o}^{\prime}$ and $A_{b y}^{\prime}$ is derived by the (P1) procedure.

\subsection{Proof of Theorem 2}

Given net-out financial system $\left(\mathcal{N}^{n e t}=\langle V, A, f\rangle, v^{*}\right)$, the net-out CCP network for $\left(\mathcal{N}^{n e t}, v^{*}\right)$ is $\mathcal{N}^{n e t}$. Take one-to-one matching $\mathcal{M}: A_{t o} \rightarrow A_{b y}$ that yields bilateral network $\mathcal{N}^{B_{n e t}}$, and denote the set of arcs derived by $\mathcal{M}$ as $A_{\text {der }}$. We can recover the net-out CCP network $\mathcal{N}^{\text {net }}$ from arbitrary $\mathcal{N}^{B_{\text {net }}}$ by undertaking arc separation on each arc $a \in A_{d e r}$ with an additional set of vertices $V_{a d d}$, and then, by undertaking vertex contraction on all the vertices in $V_{a d d}$ to have one vertex, which is interpreted as the CCP.

Proof of (i)

For given $\mathcal{N}^{B_{n e t}}=\left\langle V^{\prime}, A^{\prime}, f^{\prime}\right\rangle$, take two matched $\operatorname{arcs} a, a^{\prime} \in A^{\prime}$. Note that $f(a)=$ $f\left(a^{\prime}\right)$. Adopt arc separation for each $a, a^{\prime}$ by adding new vertices $v, v^{\prime}$. According to Lemma 1, this increases the total liquidity needs in the bad environment by $2 f(a)$. Then, adopt vertex contraction on $v, v^{\prime}$. According to Lemma 2, this decreases the total liquidity needs in the bad environment by at most $f(a)$. Thus, the combination of arc separation and vertex contraction regarding the two arcs strictly increases the total liquidity needs in the bad environment. Observe that this applies for two arbitrary matched arcs.

\section{Proof of (ii)}

The result is immediately apparent from Lemmas 1 and 2, which state that both arc separation and vertex contraction never strictly increase the total liquidity needs in the good environment.

\subsection{Proof of Theorem 3}

Given basic net-out financial system $\left(\mathcal{N}^{n e t}=\langle V, A, f\rangle, v^{*}\right)$ with $J \geq 2$ triangles, denote each triangle with $j=1,2, \ldots, J$. Then, from Lemmas 1 and 2, we obtain

(i') $x^{\max }\left(\mathcal{N}^{n e t}\right)=2 J f^{m}$ and

(ii') $x^{\min }\left(\mathcal{N}^{\text {net }}\right)=f^{m}$.

Take an arbitrary bilateral network $\mathcal{N}^{B}$ for $\left(\mathcal{N}^{n e t}, v^{*}\right)$. Observe that $\mathcal{N}^{B}$ consists of $X$ number of mutually isolated cycles with $1 \leq X \leq J$. Denote a bilateral network with $X$ cycles as $\mathcal{N}_{X}^{B_{\text {net }}}$, for which we explicitly derive liquidity needs as follows. 
(i”) $x^{\max }\left(\mathcal{N}_{X}^{B_{\text {net }}}\right)=(2 J-X) f^{m}$ and

(ii”) $x^{\min }\left(\mathcal{N}_{X}^{B_{\text {net }}}\right)=X f^{m}$.

Part (i") is evident when we observe that exactly one vertex in each cycle is exempt from inputting $f^{m}$ while the total number of the vertices is $2 J$. For part (ii"), we observe that exactly one vertex in each cycle is inputted in $f^{m}$. Combining the results completes our proof.

\subsection{Proof of Theorem 4}

For a given financial system, take arbitrary bilateral network $\mathcal{N}^{B}$. We derive a network $\mathcal{N}^{B_{n e t}}$ from $\mathcal{N}^{B}$ by the (P1) procedure. Then, we derive $\mathcal{N}^{B}$ from $\mathcal{N}^{B_{\text {net }}}$ by applying a combination of arc separation and vertex contraction, and cycle addition. As the proof of Theorem 2 shows, the combination of arc separation and vertex contraction strictly increases the liquidity needs in the bad environment. In addition, Lemma 3 shows that cycle addition strictly increases the liquidity needs in the bad environment. Since the central netting effect is viewed in the reverse direction, the central netting effect is strictly positive in the bad environment.

\subsection{Proof of Theorem 5}

Consider a one-to-one matching that derives a bilateral network for given $\left(\mathcal{N}, v^{*}\right)$. Let an $\operatorname{arc}\left(v, v^{\prime}\right)$ is derived by the matching, from a pair of vertices $\left\{\left(v, v^{*}\right),\left(v^{*}, v^{\prime}\right)\right\}$. When each of $\left(v, v^{*}\right)$ and $\left(v^{*}, v^{\prime}\right)$ is included in some offsettable pair, we call $\left(v, v^{\prime}\right)$ an offsettable arc, otherwise, we call a non-offsettable arc.

\section{Proof of (i)}

For each $V_{k}, k=1,2, \ldots, 2 K$, let $v_{k} \in V_{k}$ denote a vertex that is connected to hypothetical entity $v^{*}$, which we call a bridge vertex. For example, for $\mathcal{N}^{B_{\text {net }}}$ and $\mathcal{N}^{B}$ shown in Fig. 26, vertex $v_{i}$ is a bridge vertex. Each $v_{k}$ is included in an isolated cycle in $\mathcal{N}^{B_{\text {net }}}$, but has an additional pair of arcs with each weight $f^{m}$ in $\mathcal{N}^{B}$. It means that in the bad environment, the liquidity needs decrease by at most $f^{m}$ from $\mathcal{N}^{B}$ to $\mathcal{N}^{B_{n e t}}$ with respect to each $v_{k}, k=1,2, \ldots, 2 K$. In total, the liquidity needs decrease by at most $2 K f^{m}$. The value is actually attained for a following bilateral network. For given $\left(\mathcal{N}, v^{*}\right)$, take a bilateral network such that the relevant one-to-one matching generates a cycle in which all bridge vertices $\left\{v_{k}\right\}_{k=1,2, \ldots, 2 K}$ and at least one vertex $v \notin\left\{v_{k}\right\}_{k=1,2, \ldots, 2 K}$ are included. An example bilateral network is shown on the middle-right in Fig. 28. For such a bilateral network, it is immediately apparent that the liquidity needs decrease by $2 K f^{m}$ in the bad environment.

Regarding the opposite bound, the liquidity needs decrease by at least $K f^{m}$ in the bad environment, which is attained by a bilateral network, whereby each two isolated cycles in $\mathcal{N}^{B_{\text {net }}}$ are connected with each other in the corresponding $\mathcal{N}^{B}$, as shown in the lower row in Fig. 28. The statement is verified as follows. Consider a bilateral network for $\left(\mathcal{N}, v^{*}\right)$, in which there is no cycle that includes both an offsettable and non-offsettable arc. Take a cycle with $k^{\prime}$ number of arcs, in which every arc is an offsettable arc. In the corresponding bilateral network $\mathcal{N}^{B_{n e t}}$ for $\mathcal{N}^{B}$, the (P1) 
procedure removes a cycle so that each of the bridge vertices is now included only in a separated cycle. This reduces the relevant liquidity needs in the amount of $\left(k^{\prime}-1\right) f^{m}$. When there are $2 K$ isolated cycles (equivalently, $2 K$ bridge vertices), and $k^{\prime \prime}$ number of cycles in which every arc is an offsettable arc, then the total liquidity needs decrease by $\left(2 K-k^{\prime \prime}\right) f^{m}$. It is immediate that the smallest possible value of $\left(2 K-k^{\prime \prime}\right) f^{m}$ is attained when $k^{\prime \prime}=K$. Next, consider a bilateral network for $\left(\mathcal{N}, v^{*}\right)$, in which there is at least one non-offsettable arc, and there are $k^{\prime \prime \prime}$ number of bridge vertices. In the corresponding bilateral network $\mathcal{N}^{B_{n e t}}$ for $\mathcal{N}^{B}$, the (P1) procedure let each of the bridge vertices be disconnected from the relevant cycle. This reduces the relevant liquidity needs in the amount of $k^{\prime \prime \prime} f^{m}$. Thus, the total liquidity needs decrease by at least more than $K f^{m}$.

\section{Proof of (ii)}

Consider a bilateral network $\mathcal{N}^{B}$ for $\left(\mathcal{N}, v^{*}\right)$, for which cycle addition is not relevant for the corresponding (P1) procedure. For such $\mathcal{N}^{B}$, there is no cycle in which every arc is an offsettable arc. Each step of the (P1) procedure effectively lets each isolated cycle be disconnected from some other cycle. Thus, for corresponding $\mathcal{N}^{B_{n e t}}$, we obtain $x^{\min }\left(\mathcal{N}^{B}\right)-x^{\min }\left(\mathcal{N}^{B_{n e t}}\right)=-\Sigma_{1}^{2 K} \min \left(f^{k}, f^{m}\right)$ from part (ii) of Lemma 2.

Then, consider a bilateral network $\mathcal{N}^{B}$ for $\left(\mathcal{N}, v^{*}\right)$, for which cycle addition is relevant for the corresponding (P1) procedure. For such $\mathcal{N}^{B}$, there is at least one cycle in which every arc is an offsettable arc. Take such a cycle, which we denote as cycle $c$. Take another cycle $c^{\prime}$ that includes a non-offsettable arc. For $\mathcal{N}^{B}$, we obtain another bilateral network $\mathcal{N}^{B^{\prime}}$ by adopting cycle merge on $c$ and $c^{\prime}$. Concretely, take arbitrary arc $\left(v_{a}, v_{b}\right)$ of cycle $c$, and a non-offsettable arc $\left(v_{c}, v_{d}\right)$ of cycle $c^{\prime}$. Note that the weight for each of $\left(v_{a}, v_{b}\right)$ and $\left(v_{c}, v_{d}\right)$ is $f^{m}$. Let $\left\{\left(v_{a}, v_{b}\right),\left(v_{c}, v_{d}\right)\right\}$ be replaced with a pair of $\operatorname{arcs}\left\{\left(v_{a}, v_{d}\right),\left(v_{c}, v_{b}\right)\right\}$, for which each weight is set as $f^{m}$. The derived network is apparently a bilateral network for $\left(\mathcal{N}, v^{*}\right)$. Observe that the $(\mathrm{P} 1)$ procedure for $\mathcal{N}^{B^{\prime}}$ yields $\mathcal{N}_{\text {net }}^{B}$, which is the same network derived by the (P1) procedure for $\mathcal{N}^{B}$. Thus, we are suffice to compare $x^{\min }\left(\mathcal{N}^{B}\right)$ to $x^{\min }\left(\mathcal{N}^{B^{\prime}}\right)$. It is immediately apparent that $x^{\min }\left(\mathcal{N}^{B}\right)>x^{\min }\left(\mathcal{N}^{B^{\prime}}\right)$, since the relevant operation effectively connects cycles. Observe that we can repeatedly adopt cycle merge until there is no cycle in which every arc is an offsettable arc. This proves that the maximum negative effect is attained as $-\Sigma_{1}^{2 K} \min \left(f^{k}, f^{m}\right)$.

\section{Proof of (ii')}

From the argument in the proof of (ii), we are suffice to focus on bilateral networks for $\left(\mathcal{N}, v^{*}\right)$, in which there is no cycle that includes both an offsettable and nonoffsettable arc. For such a bilateral network, consider the relevant (P1) procedure. Suppose the relevant reverse of cycle addition removes a cycle to form $k^{\prime}$ number of isolated cycles. Then, the relevant liquidity needs increase by $\left(k^{\prime}-1\right) f^{m}$, according to Lemma 3 . Since there are $2 K$ number of isolated cycles for arbitrary bilateral network for given $\left(\mathcal{N}^{n e t}, v^{*}\right)$, the total liquidity needs increase by at least $K f^{m}$, whereby the value is attained with bilateral networks for $\left(\mathcal{N}, v^{*}\right)$, in which every isolated cycle is connected to another isolated cycle. 


\subsection{Proof of Theorem 6}

\section{Proof of (i)}

The result is immediately apparent by combining part (i) of Theorem 3 and part (i) of Theorem 5.

\section{Proof of (ii)}

First, we examine the central netting effect. Part (ii) of Theorem 5 ensures that the lower bound of the central netting effect for the class specified in the current theorem is also $-\Sigma_{1}^{2 K} \min \left(f^{k}, f^{m}\right)$. The proof shows that the value is attained in a bilateral network, such that all the obligations relevant to the hypothetical entity form a cycle, in which every isolated cycle is connected to the cycle. Furthermore, according to part (ii) of Theorem 3, the lower bound of the central routing effect is zero. The proof shows that the value is attained between a bilateral network examined above and the corresponding net-out CCP network.

\section{Proof of (ii')}

Again, we first examine the central netting effect. According to part (ii') of Theorem 5, the smallest negative effect for the class specified in the current theorem is also $K f^{m}$. The proof shows that the value is attained in a bilateral network such that every isolated cycle is paired to another isolated cycle, and each triangle turns out to be a pair of obligations between the relevant two vertices. Then, for the central routing effect, according to part (ii) of Theorem 3, the largest positive effect is $(J-1) f^{m}$, and the proof shows that the value is attained between a bilateral network examined above and the corresponding net-out CCP network. Since it is assumed that $J \leq K$, the smallest possible negative total effect is derived as $-f^{m}$.

\section{References}

Abruzzo N, Park YH (2016) An empirical analysis of futures margin changes: determinants and policy implications. J Financ Serv Res 49(1):65-100

Ahuja RK, Magnanti TL, Orlin JB (1993) Network flows. Prentice Hall, Englewood Cliffs

Bliss RR, Kaufman GG (2006) Derivatives and systemic risk: netting, collateral, and closeout. J Financ Stab 2(1):55-70

Bliss RR, Steigerwald RS (2006) Derivatives clearing and settlement: a comparison of central counterparties and alternative structures. Econ Perspect 30(4):22-29

Brunnermeier MK, Pedersen LH (2009) Market liquidity and funding liquidity. Rev Financ Stud 22(6):22012238

Domanski D, Gambacorta L, Picillo C (2015) Central clearing: trends and current issues. BIS Q Rev

Duffie D, Zhu H (2011) Does a central clearing counterparty reduce counterparty risk? Rev Asset Pricing Stud 1(1):74-95

Galbiati M, Soramäki K (2012) Clearing networks. J Econ Behav Organ 83(3):609-626

Hayakawa H (2014) Complexity of payment network. https://doi.org/10.2139/ssrn.2448552

Hayakawa H (2016) Characterization of lower bound and upper bound of required settlement fund under real-time gross settlement. https://doi.org/10.2139/ssrn.2659975

Jackson JP, Manning MJ (2007) Comparing the pre-settlement risk implications of alternative clearing arrangements. Bank of England Working Paper No. 321

Miglietta A, Picillo C, Pietrunti M (2015) The impact of ccps' margin policies on repo markets. BIS Working Papers (515)

Murphy D, Vasios M, Vause N (2014) An investigation into the procyclicality of risk-based initial margin models. Bank of England, Financial Stability Papers (29) 
Pirrong C (2009) The economics of clearing in derivatives markets: netting, asymmetric information, and the sharing of default risks through a central counterparty. Working Paper, University of Houston

Rennison J, Meyer G, Stafford P (2016) Derivatives traders forced to provide \$27bn collateral post-Brexit, Finanical Times, November 172016

World Bank (2013) Global financial development report 2013: rethinking the role of the state in finance. World Bank, Washington, DC 